\title{
Welche plebiszitären Instrumente könnten wir brauchen? Einige systematische Überlegungen.
}

\author{
Werner J. Patzelt
}

\section{A. Plebiszitäre „Instrumente“ oder „Elemente“?}

Es ist gut, dass Demokratie inzwischen allenthalben als wertvoll und erstrebenswert gilt. Einmal bestehend, legt Demokratie dann die Frage nahe, ob es vom Guten nicht ein wenig mehr sein dürfe. Hat man also repräsentative Demokratie, zu verstehen als gleichsam ,indirekte“ Demokratie, dann bestünde der nächste Schritt darin, zur noch besseren Form der Demokratie überzugehen, nämlich zur „direkten“ Demokratie. Dafür fallen einem schnell mancherlei Instrumente ein: Direktwahl nicht nur von Abgeordneten, sondern auch möglichst vieler Amtsträger (zumindest der Bürgermeister, Landräte, Ministerpräsidenten und des Staatsoberhaupts); Gesetzgebung nicht nur durch Parlamente, sondern auch durchs Volk; Volksabstimmungen auch über Sachfragen; und schließlich umfangreiche Möglichkeiten bürgerlicher Mitwirkung an Politik wie Zukunftswerkstätten und Planungszellen. ${ }^{1}$ Von einer - selten vertretenen - „stark direktdemokratischen“ Position aus sollen durch solche Instrumente Volksvertretungen im Grunde überflüssig werden, damit das Volk nicht mehr mediatisiert ${ }^{2}$ und Demokratie fortan „,vollendet“ sei. In einer viel häufiger vertretenen ,gemäßigt direktdemokratischen“ Position soll repräsentative Demokratie hingegen nicht ersetzt, sondern durch plebiszitäre Instrumente einfach verbessert werden.

1 Siehe P.C. Dienel, Die Planungszelle. Der Bürger als Chance, 5. Aufl. 2002.

2 „Mediatisierung“ sollte, obwohl in der Kommunikationswissenschaft nicht selten gleichbedeutend gebraucht, nicht mit „Medialisierung“ gleichgesetzt werden. Der erstgenannte Begriff bezeichnet, was mit dem Ende des alten deutschen Reiches den bis dahin reichsunmittelbaren, allein dem Kaiser unterstellten Reichstädten, Reichsgrafen usw. widerfuhr: Sie wurden den nunmehr völlig souverän gewordenen großen Landesherren unterstellt, die ihrerseits den Deutschen Bund bildeten. Zwischen dem Bund insgesamt und ihnen (lat. ,in medio“, d.h. in der Mitte) standen nun etwa die Könige von Preußen und Sachsen, von denen die bis dahin reichsunmittelbaren Stände somit „mediatisiert“ wurden. Eben dies bewirken - in demokratietheoretischer Perspektive - auch Volksvertreter, die ihre eigenen Entscheidungen zwischen die Willensbekundungen des Volkes und den etwa in Gesetzesbeschlüssen fassbaren Staatswillen setzen. 
Dann wird es vorteilhaft sein, erst einmal jene Probleme zu benennen, die durch plebiszitäre Systemverbesserungen behoben werden sollen, und sodann zu prüfen, welches besondere plebiszitäre Instrument zur Behebung welchen Problems auch geeignet sein mag. Umsichtigem Denken wird es dabei dienen, wenn man die Rede von plebiszitären „Elementen“ hinterfragt. Sie hat immerhin recht Unverbindliches an sich, weist sie doch nur darauf hin, dass es im Warenlager der Demokratie noch weitere „Elemente“ als nur die „repräsentativen“ gäbe, nämlich ,plebiszitäre“; und die solle man nicht als Ladenhüter verstauben lassen, sondern seinem demokratisches System einbauen.

Ganz anders richtet hingegen die Rede von „plebiszitären Instrumenten“ die Aufmerksamkeit aus. Ein Instrument dient schließlich nicht als Zierstück, sondern dazu, eine Aufgabe zu erledigen oder ein Problem zu beheben. Es anzuwenden, wo nicht nötig, ist deshalb unverständig; und ein Instrument einzusetzen, dessen Nebenwirkungen mehr Schaden stiften, als das ganze Instrument nutzt, wirkt wenig vernünftig. Wer also von ,plebiszitären Instrumenten“ spricht, erlegt sich eine gründliche Klärung jener Konstruktionsprobleme einer repräsentativen Demokratie auf, die zur plebiszitären Bewältigung anstehen, und verpflichtet sich zur Heranziehung funktionslogischen sowie historischen Wissens um die Risiken und Nebenwirkungen jeweils empfohlener Instrumente. Hingegen gibt die Rede von ,plebiszitären Elementen“ nur unverbindliche Verschönerungshinweise und insinuiert, ein politisches System könne gleichsam additiv weiterentwickelt werden: Man fügt eben, entlang dessen demokratischer Leitidee, weitere (direkt-) demokratische „Elemente“ hinzu, womit Demokratie noch schöner und besser wird. So einfach wird das aber nicht sein.

\section{B. Repräsentation, Demokratie, plebiszitäre Instrumente: Wie hängt das alles zusammen?}

\section{Die Betrachtungsperspektive}

Alle Überlegungen müssen ihren Ausgang vom rechten Verständnis repräsentativer Demokratie nehmen. Zu diesem gehört sehr wohl empirisches und zeitgeschichtliches Wissen um die Vorzüge repräsentativer Demokratie. Es wird ja nicht rein zufällig sein, dass mit demokratisch gewählten Parlamenten ausgestattete Regime weltweit die stabilsten, am wenigsten von Rückfällen in Diktatur bedrohten und obendrein die wirtschaftlich wie gesellschaftsintegrierend erfolgreichsten politischen Systeme sind. Auch sind sie, trotz aller Politikverdrossenheit, jene politischen Ordnungen, die sich noch am meisten auf freiwilligen Ge- 
setzesgehorsam sowie auf grundsätzliche Akzeptanz seitens ihrer Bürger verlassen können. Und unter ihnen scheinen die parlamentarischen Regierungssysteme, die keine Direktwahl des Staats- oder Regierungschefs kennen, in sämtlichen dieser Merkmale den präsidentiellen und zumal semipräsidentiellen Regierungssystemen mit Direktwahl des Staats- und Regierungschefs überlegen zu sein. ${ }^{3}$ Ausnahme ist allein das präsidentielle Regierungssystem der USA, wo diese Form der konstitutionellen ,republikanischen Monarchie“ im späten 18. Jh. erfunden und erstmals praktiziert wurde.

Doch mit dem völlig zutreffenden Hinweis auf die historische Bewährung und erprobte Überlegenheit repräsentativer Demokratie ist ja noch nicht gesagt, dieser Regimetyp funktioniere ohne jedes strukturelle Problem. Ebensowenig zwingt die Einsicht in den Wert repräsentativer Demokratie zur Vermutung, kein einziges plebiszitäres Instrument könne bei der Lösung einiger struktureller Probleme repräsentativer Demokratie helfen. Die Scharmützel der Art „Hie Welf, hie Waibling“ kann man also jenen überlassen, welche die Diskussion um den möglichen Nutzen plebiszitärer Instrumente lieber wie eine Glaubensfrage behandeln. Besser ist es, auf dieses Thema aus dem Blickwinkel eines Ingenieurs zu blicken, der seine repräsentative Maschinerie dank langer Vertrautheit sehr schätzt, gerade deshalb aber ihre Funktionsprobleme nicht verkennt und Bewährtes eben verbessern will.

\section{Das Spannungsverhältnis zwischen Repräsentation und Demokratie}

Ein historisch aufgeklärtes Verständnis repräsentativer Demokratie kommt um die Feststellung nicht herum, dass Repräsentation und Demokratie zunächst einmal nichts miteinander zu tun haben. Sieht man von den - räumlich und zeitlich Episode bleibenden und obendrein ganz repräsentationsfeindlichen - griechischen Stadtstaaten $a b$, geht nämlich Repräsentation der Demokratie voraus und funktionierte jahrhundertelang auch ganz ohne Demokratie. Ein intuitives Grundverständnis dessen wirkt überall nach, wo sich um der Demokratie willen Unbehagen an Repräsentation äußert und man deshalb nach der Fortentwicklung einer „bloß“ repräsentativen Demokratie hin zur „eigentlichen“, nämlich nicht nur durch Repräsentanten vermittelten, Demokratie verlangt.

Typisch für die europäische Geschichte ist zumal die ständische Repräsentation. Bei ihr wirken die rechtlich wie faktisch realen Machtträger eines Gebiets

3 Siehe etwa J. Linz, The failure of presidential democracy, Baltimore u.a., 1994. 
mit ineinander greifenden Herrschaftsbefugnissen zusammen, wobei teils Territorien (etwa das Herzogtum X), teils Korporationen (wie die Universität Y oder die Bürgerschaft der Stadt Z) vertreten sein konnten. Die europäischen Versammlungen von Land- und Reichsständen - aus denen später, am deutlichsten im Fall der englischen Houses of Parliament, die modernen Parlamente hervorgehen sollten - waren die Stätte solcher Repräsentation. ${ }^{4}$ Liberale Repräsentation stellte deren institutionelle Form in den Dienst einer tiefgreifend veränderten Leitidee: Repräsentanten - sofern sie nicht einfach sich selbst „vertraten“ wie ein Fürst auf einem Reichstag - sollten nicht länger bindende (,imperative“) Mandate der sie entsendenden Stände haben, sondern mit „freiem“ Mandat ausgestattet sein, um nämlich durch die genau dann mögliche Mannschaftsbildung in der Vertretungskörperschaft Gegenmacht zur Exekutivgewalt aufbauen zu können. ${ }^{5}$ Damit solche Mannschaftsbildung nicht eigensüchtigen Zwecken diene, sollten sich Abgeordnete außerdem nicht länger als Vertreter allein der sie Entsendenden, sondern der ganzen Bürgerschaft und des gesamten Gemeinwesens verstehen. Das wurde später ,virtuelle Repräsentation“ genannt.

Die demokratische Repräsentation, seit der amerikanischen und der Französischen Revolution auf Siegeszug, fügte dem noch die freie, da geheime, und nach etlichen Jahrzehnten auch noch die gleiche, allgemeine und unmittelbare Wahl der Repräsentanten hinzu. Periodische Wahlen in nicht zu großen Abständen institutionalisierten dann noch temporale Gewaltenteilung über den sogenannten „Wiederwahlmechanismus“: Wer sein Amt erringen oder verlängert bekommen will, kann während seiner Amtszeit nicht zu weit oder zu lange von dem abweichen, was seine Wähler zu akzeptieren bereit sind. ${ }^{6}$ Periodische Wahlen aber ließen den bereits als „Parlamentsclubs“ entstandenen parlamentarischen Parteien noch eine zweite, im Lauf der Zeit immer wichtiger werdende Wurzel zuwachsen: Sie führten nämlich zur Entstehung von Parteien in ihrer Rolle als Wahlvereine. Sobald dann noch Kandidaten und Abgeordnete verstanden hatten, dass sie ihr Mandat vor allem, ja allein mit Unterstützung eines solchen „Wahlvereins“

4 Moderne Ableger einer - freilich vom Politisch-Ständischen ins Berufsständische gewandelten - ständischen Repräsentation sind jene Wirtschafts- und Sozialräte, die manchen internationalen Organisationen, doch etwa auch dem französischen politischen System und dem supranationalen System der EU beigegeben sind. Im bundesdeutschen Parlamentarismus lebte derlei jahrzehntelang im 1998 durch Volksentscheid abgeschafften Bayerischen Senat fort.

5 Solche parlamentarische Club- und Fraktionsbildung ist die erste von zwei Wurzeln moderner Parteien; siehe W. Steffani, Zur Vereinbarkeit von freiem Mandat und Fraktionsdisziplin, in: ZParl 12 (1981), S. 109-122.

6 Siehe W.J. Patzelt, Einführung in die Politikwissenschaft, 6. Aufl. 2007, S. 274 ff. 
(wieder-) erlangen konnten, begannen sie sich an die Spitze jener Parteiorganisationen zu setzen, die für ihre (Wieder-) Wahl ausschlaggebend waren. Auf diese Weise entstand gemeinsam mit demokratischer Repräsentation der von starken und durch Abgeordnete geführte - Parteien getragene Staat.

Ein gemeinsamer Nenner aller Repräsentationssysteme, und auch demokratischer Repräsentation, besteht nun darin, dass derlei unvermeidlich zur Veranstaltung von Eliteangehörigen gerät. Allenfalls der Zugang zur ,politischen Klasse“ wird unter den Bedingungen einer Demokratie sozial geöffnet. ${ }^{7}$ Zugleich geht seit den griechischen Stadtstaaten mit der Vorstellung von Demokratie auch noch die Idee einher, es sei wünschenswert, die Trennung zwischen Regierten und Regierenden, zwischen dem Volk und seinen Vertretern aufzuheben. Deshalb entsteht zusammen mit demokratischer Repräsentation oft auch ein demokratietheoretisches Ärgernis: Sobald man auf Repräsentation setzt, lässt sich nicht mehr der höchst attraktiv in der Fluchtlinie demokratischen Denkens liegende Gedanke verwirklichen, die für alle Arbeitsteilung grundlegende Trennung zwischen „Prinzipalen“ und „Agenten“ solle aufgehoben sein. ${ }^{8}$

Natürlich ergibt es wenig Sinn, demokratische Repräsentation einzuführen, wenn man nicht darauf setzen will, dass hauptamtliche Politiker sich kontinuierlicher, tiefer und unter größerem Leistungsdruck mit zu lösenden Problemen befassen werden als Bürger, die derlei fallweise unternehmen. Ebensowenig ergibt die Verbindung von Repräsentation mit Demokratie Sinn, wenn man nicht umgekehrt auch auf die Urteilsfähigkeit der Repräsentierten zu setzen bereit ist. Dann freilich lässt sich jenes demokratietheoretische Grundärgernis gerade nicht mehr mit dem Appell aus der Welt schaffen, man möge es mit der Demokratie nicht zu weit treiben: Allenfalls für die Wahl von Repräsentanten reiche die Klugheit von Bürgern, nicht aber für Sachentscheidungen. Folglich wird man mit jenem Grundärgernis einfach leben müssen und von ihm Mal um Mal daran erinnert werden, dass Repräsentation und Demokratie durchaus nicht zwei Seiten

7 Unter den Begriff der ,politischen Klasse“ fallen jene Personen, die in einem Gemeinwesen nicht nur für, sondern großenteils auch von der Politik leben und diesbezüglich gemeinsame Professionsinteressen entwickeln. Er ist also ein analytischer Begriff, der im Folgenden niemals abwertend zu verstehen ist.

8 Die Prinzipal-Agent-Theorie, nicht selten auch Delegations- oder Agenturtheorie genannt, ist in der Neuen Institutionenökonomik entstanden und hat in den letzten Jahren das repräsentationstheoretische Denken stark geprägt. Ihr geht es um die Beziehungen zwischen faktischen oder fiktiven Auftraggebern („Prinzipalen“) und deren - unterschiedlicher Aufgaben wegen eingestellten - Beauftragten (,Agenten“). Blickt man auf Politik, so geht es bei der Delegationstheorie um das Verhältnis zwischen dem Volk und seinen Politikern. Hierzu siehe etwa F. Gilardi/D. Braun, Delegation aus der Sicht der Prinzipal-Agent-Theorie, in: PVS 43 (2002), 147 ff. 
derselben Medaille, sondern ganz unterschiedliche Ausgestaltungsprinzipien eines politischen Systems sind, die zwar sehr gut, aber nicht fugenlos miteinander verbunden werden können.

Eben an jene „Fugen“ erinnert immer wieder die Gegenüberstellung von „,indirekter“ und ,direkter“ Demokratie sowie die Attraktivität von Appellen dahingehend, die ,indirekte Demokratie“ solle doch so weit wie möglich mit ,direktdemokratischen Elementen“ angereichert werden. Doch selbst wenn man eine solche „stark direktdemokratische“ Position nicht teilt, sondern von einer ,gemäßigt direktdemokratischen" Position aus den Eigenwert demokratischer Repräsentation nicht bestreitet, lassen sich mindestens zwei Folgeprobleme einer Verbindung von Repräsentation mit Demokratie nicht übersehen.

Erstens führt das - nicht erst, aber erst recht - mit Demokratie einhergehende Aufkommen von periodischen Wahlen zur Entstehung von Wahlvereinen und später von Parteien. ${ }^{9}$ Dann aber kann nicht mehr, und schon gar nicht im gemäß seiner Funktionslogik mannschaftsbildenden parlamentarischen Regierungssystem, ${ }^{10}$ die geschichtlich vorgängige und einer ,indirekten Demokratie“" so sehr zupass kommende liberale Fiktion aufrechterhalten werden, jeder Abgeordnete sei ein „Vertreter des ganzen Volkes““. ${ }^{11}$ Tatsächlich wurde noch jeder Kandidat von einem - oft sogar beträchtlichen - Teil der Bürgerschaft als ihr Vertreter $a b$ gelehnt. Dies vor Augen, verschiebt man die Überlegungen oft ins hier Nebensächliche und beklagt eine zu große „Parteiorientierung“ der Parlamentarier, obwohl doch die Wähler selbst ganz wesentlich nach ihrer - freilich flottierenden Parteiorientierung entscheiden. Tatsächlich rührt das ganze Problem nur vom Missverständnis her, dass es überhaupt auf die Vertretung gleich des ganzen Volkes hinauslaufen müsse, wenn man in Wirklichkeit die Bürger eines Wahlkreises oder die Wähler einer Partei vertritt.

Um doch noch die „Vertretung des ganzen Volkes“ denkbar zu machen, behilft man sich nicht selten mit einer statistischen Vorstellung: Eine demokratische Vertretungskörperschaft müsse ein ,repräsentativer Querschnitt“ der Bevölkerung sein. Am sichersten garantiert das freilich nicht ein Quotenverfahren bei der Kandidatennominierung, sondern ein Losverfahren anstelle einer Wahl, wie es denn auch - allerdings recht unzulänglich - die jeder Repräsentation ab-

9 Zur anderen, früheren Wurzel von Parteien siehe die Fn. 3.

10 Vgl. S.S. Schüttemeyer, Funktionserfüllung im Fraktionenparlament, in: Informationen zur politischen Bildung 295 (2007), 40 ff.

11 So aber beschreiben das Abgeordnetenmandat nicht wenige Verfassungen; siehe etwa Art. 38 Abs. 1 GG: „Die Abgeordneten ... sind Vertreter des ganzen Volkes, an Aufträge und Weisungen nicht gebunden und nur ihrem Gewissen unterworfen“. 
holden Athener bei der Besetzung ihrer Ratsversammlung und ihrer Volksgerichte praktizierten. ${ }^{12}$ Will man Amtsinhaber auslosen, darf man aber nicht auf eine besondere, sich vom Bevölkerungsdurchschnitt abhebende politische Begabung der Volksvertreter hoffen und entzieht damit dem Repräsentativsystem eine wichtige Rechtfertigung. Wahlen wiederum, die sehr wohl Selektionsdruck hin auf besondere politische Begabung schaffen können, erzeugen gerade keinen „repräsentativen Querschnitt“ aus der Bevölkerung: Nicht jeder ist gleich willig, sich auf eine politische Laufbahn einzulassen; nicht jeder ist gleich tüchtig, sie zu bestehen; und nicht jeder kann soviel Sozial- und Finanzkapital mobilisieren, wie zum Sieg erforderlich wäre.

Gewiss kann sich kein größeres Volk an gemeinsamer Stätte zur Willensbildung und Entscheidungsfindung versammeln. Also muss - falls man Demokratie will - ein wie immer verzerrter „Querschnitt des Volkes“ an die Stelle des ,eigentlich“ zu versammelnden ganzen Volkes treten, der dann das durchaus nicht gegenwärtige Volk in gewisser Weise eben doch gegenwärtig macht. Versteht man demokratische Repräsentation genau dahingehend, ${ }^{13}$ wird man keine guten Gründe zur Abwehr folgender Anschlussforderung haben: Wo immer institutionelle Erfindungen und technische Möglichkeiten derlei Repräsentation als bloßen „Notbehelf“ überflüssig machen können, sollten sie auch umfänglich genutzt werden. Dieses ,stichprobenartige“ Verständnis von politischer Repräsentation ${ }^{14}$ liegt dem Alltagsdenken vieler Bürger zugrunde, prägt einen Großteil populärer Kritik an der Zusammensetzung von Parlamenten und bringt ganz intuitiv die „Korrekturmöglichkeiten“ plebiszitärer Instrumente vor Augen.

Zweitens muss jene politische Stimmungslage und Präferenzverteilung, die am Wahltag über die fortan bestehenden parlamentarischen Stärkeverhältnisse entschied, ja nicht andauern. Tatsächlich pflegen demokratisch ins Amt gekommene Regierungen schon bald nach Amtsantritt an öffentlicher Unterstützung einzubüßen. Dann ist ein Parlament, nach Stimmungs- und Meinungsumschwüngen in

12 Gerade der Demokratie willen setzten sie also lieber auf den Zufall oder den Willen der Götter als auf Wahlen, für Erfolg bei welchen immer schon Vernetzung, Ansehen und letztlich Wirtschaftsmacht wesentlich sind.

13 Man kann demokratische Repräsentation aber alternativ wie unten beschrieben verstehen: Der „Wiederwahlmechanismus“ macht Repräsentanten einfach besonders responsiv.

14 Der vernünftige Kern dieser Repräsentationsvorstellung besteht darin, dass die Kommunikations- und Interaktionsnetze der Repräsentanten tatsächlich in die ganze Gesellschaft hineinreichen sollten, damit von überall her Responsivität eingefordert und überall hin kommunikative politische Führung ausgeübt werden kann. Am besten gelingt derlei, wenn die Repräsentanten wirklich aus den verschiedensten Bereichen und Schichten der Gesellschaft stammen oder wenigstens überall hin Kontakte aufgebaut haben. 
der Bevölkerung, durchaus nicht mehr „repräsentativ“ im demoskopischen Sinn. Kommt es in dieser Lage zu parlamentarischen Entscheidungen gegen demoskopisch feststellbare Mehrheiten im Volk, wird nicht selten bezweifelt, dass solche Entscheidungen ,demokratische Legitimität“ besäßen. ${ }^{15}$ Erst recht gilt das, wenn man bei der Parlamentsmehrheit ein systematisches „Anregieren gegen das Volk“ zu erkennen glaubt. ${ }^{16}$ Drei Lösungsmöglichkeiten für dieses Problem bieten sich dann an. Erstens kann man ein Recht auf Abberufung von Abgeordneten seitens ihrer Wähler bzw. Nominierungsgremien einführen. Zweitens kann man - über ein Selbstauflösungsrecht von Parlamenten hinaus - an die Ansetzung von Neuwahlen aufgrund einer Volksabstimmung denken. Drittens kann man auf Möglichkeiten des Volkes sinnen, seinen Vertretern die eigenen Themen, Argumente und Präferenzen Mal um Mal aufzuzwingen, falls es sich von seinen Politikern unzulänglich repräsentiert fühlt. Eben das ist der „Sitz“ von Volksanträgen und Vorlageninitiativen, von Volksgesetzgebung und fakultativen Gesetzesreferenden in einer repräsentativen Demokratie.

\section{Der Mehrwert repräsentativer Demokratie}

Nun steht dem verbreiteten und ganz zutreffenden Eindruck, dass sich Repräsentation und Demokratie nicht ohne Weiteres vertragen, der kaum minder verbreitete und ebenfalls ganz zutreffende Eindruck gegenüber, dass gerade die Verbindung von Repräsentation und Demokratie besonders leistungsfähige Regierungssysteme schafft. Die Synergieeffekte von Repräsentation und Demokratie scheinen ihrerseits mit Pluralismus und der Wertschätzung von Konflikten zu tun zu haben. Pluralismus wird nämlich nirgends deutlicher als in Wahlkämpfen, und in friedlichen Bahnen gehaltener Konflikt wird nirgendwo sichtbarer als im parlamentarischen Gegenüber von Regierungsmehrheit und Opposition. Der Mehrwert von pluralistischen Konflikten, auszutragen unter den Bedingungen des Wiederwahlmechanismus, besteht wiederum im durch sie erzeugten Druck hin auf politisches Lernen: Wer wegen der von ihm vertretenen Positionen eine Wahl nach der anderen verliert, wird entweder bald keine politisch wichtige Rolle mehr spielen oder zur Anpassung gezwungen - wie in den späten 1950er Jah-

15 Theoretisch-systematisch wäre hier von den stets problematischen Vertrauensbeziehungen zwischen „Prinzipalen“ und deren „Agenten“ zu handeln, wie sie die Delegationsbzw. Agenturtheorie in den Mittelpunkt ihrer Analysen rückt; siehe Fn. 8.

16 Vgl. W.J. Patzelt, Warum regieren Politiker gegen die Bürger?, in: R. Riedl/E. Gehmacher/W. Hingst (Hrsg.), Regieren gegen den Bürger?, 2006, S. 273-302. 
ren die SPD zu „Godesberg“ und nach 2003 die CDU zur „Sozialdemokratisierung“. Lernfähige Abgeordnete, Parteien, Regierungen und Regime werden aber bessere Chancen haben, grobe Politikfehler zu vermeiden, und sie werden sich deshalb leichter damit tun, in einem Gemeinwesen freiwilligen Gesetzesgehorsam und innengeleitete Leistungsbereitschaft auf Dauer zu stellen. Anders als Repräsentation und Demokratie sind somit Lernfähigkeit und Legitimationsfähigkeit wirklich zwei Seiten derselben Medaille.

Nun erlaubt es allerdings status quo-sichernde Macht, vielerlei „eigentlich“ bestehenden Lerndruck zu mildern, ja abzuweisen. ${ }^{17}$ Wer also den zentralen Mehrwert demokratischer Repräsentation darin erkennt, dass sie zu pluralistischen Konflikten führt, die ihrerseits die Lernfähigkeit von Politik steigern, der wird auch sicherstellen wollen, dass Repräsentanten unter Lerndruck bleiben und sich diesem nicht durch solche Macht entziehen können, die ihnen qua Amt oder faktischer Rolle nun einmal zuwächst. Kritische Medienberichterstattung, demoskopische Umfragen und freie Wahlen sind jetzt schon sehr wirksame Mittel dafür. Weitere Mittel, ein politisches System über seine Amtsträger lernwillig zu machen, können Vorwahlen sein, mit denen bereits auf die Kandidatenaufstellung wirksam Einfluss genommen wird. ${ }^{18}$ Ein zusätzliches und obendrein punktgenau einsetzbares Mittel zur Steigerung der Lern- und Legitimationsfähigkeit repräsentativer Demokratie sind plebiszitäre Instrumente. Wo sie wirkungsvoll ansetzen könnten, erschließt der folgende Gedankengang.

\section{Wo könnten plebiszitäre Instrumente einer repräsentativen Demokratie dienlich sein?}

Repräsentation ist eine Form politischer Arbeitsteilung, innerhalb welcher Repräsentanten die Herstellung und Durchsetzung allgemein verbindlicher Regeln und Entscheidungen als Dienstleistung für eine Gesellschaft erledigen. ${ }^{19}$ Wer die Sache so sieht, wird auf einer gewissen Qualitätssicherung beim Zugang zu öffentlichen Ämtern bestehen und erwarten, was Ernst Fraenkel einst in eine berühmte Formel gefasst hat: Bei Repräsentation gehe es um die „Veredelung des

17 K.W. Deutsch, Politische Kybernetik. Modelle und Perspektiven, 1969, S. 171, formulierte dies einst dahingehend, dass Machtbesitz das Privileg verleihe, nicht lernen zu müssen.

18 Siehe W.J. Patzelt, Verbessern wir unsere repräsentative Demokratie! Ein Weg: Vorwahlen in Deutschland, Thesenpapier, 2010 (erhältlich vom Verfasser).

19 Damit ist natürlich eine notwendige, keinesfalls eine hinreichende Bedingung formuliert: Repräsentation ist eine Form eines politischen Dienstleistungsverhältnisses; doch nicht jedes politische Dienstleistungsverhältnis ist Repräsentation. 
empirisch vorfindbaren Volkswillens“ hin zu jenem „hypothetischen Volkswillen“, den eine Bürgerschaft dann hätte, wenn sie sich ebenso zeitaufwendig, andauernd und gründlich über gesellschaftliche Gestaltungsaufgaben informieren könnte, wie sie das als „Prinzipal“ von der politischen Klasse als ihren „Agenten" erwartet. ${ }^{20}$

Damit ist die zentrale Bringschuld von Repräsentanten formuliert. Also droht einem Repräsentativsystem der Wegfall seiner Geschäftsgrundlage, wenn die politische Klasse binnenbezüglich wird und eher intern ihr eigenes Bestes als jenes Gemeinwohl sucht, das nur im offenen pluralistischen Streit auch mit der Bürgerschaft zu klären ist. Sofern außerdem die politischen Rekrutierungsmuster einer Gesellschaft vor allem solche Personen in die politische Klasse gelangen lassen, die sich zwar gut auf binnenbezüglichen Politikspiele und wiederwahlsichernden Medienumgang verstehen, viel weniger aber auf die sachlichen Dimensionen der ihrer Sorgsamkeit anvertrauten wirtschaftlichen, fiskalischen, technischen, sozialen, kulturellen und demographischen Zusammenhänge, ist die Grundlage demokratischer Repräsentation von vornherein ausgedünnt. Auf das zweite Problem kann man mit Veränderungen der Rekrutierungs- und Selektionsbedingungen reagieren, etwa durch Einführung von Vorwahlen. Zur Behebung des erstgenannten Problems gibt es plebiszitäre Instrumente. Deren systemkompatible Einsatzmöglichkeiten erkennt man beim Blick auf die drei konstitutiven Merkmale einer Repräsentationsbeziehung. ${ }^{21}$

Am ersten Eck des „Repräsentationsdreiecks“ steht: Die Repräsentanten handeln im Interesse der Repräsentierten und dabei responsiv. Responsivität meint dabei die Reaktionswilligkeit, Reaktionsbereitschaft, Reaktionsfähigkeit, Sensibilität und Anregbarkeit einer Person oder eines (sozialen) Systems, etwa einer Partei oder eines Parlaments. Angelpunkt von Repräsentation sind also stets die Interessen der Repräsentierten; und eine zentrale Aufgabe von Repräsentanten besteht darin, um diese Interessen genau zu wissen und sie ernstzunehmen. Dabei gehört es zur wesentlichen Leistung von Repräsentanten, in eigener Verantwortung auch latente Interessen der Repräsentierten hinter deren manifesten Interessen aufzuspüren, subjektive Interessen von objektiven, Partikularinteressen von Allgemeininteressen zu unterscheiden sowie einen klugen Kurs zwischen

20 Siehe E. Fraenkel, Die repräsentative und die plebiszitäre Komponente im demokratischen Verfassungsstaat, in: ders., Deutschland und die westlichen Demokratien, 7. Aufl. 1979, S. $113 \mathrm{ff}$.

21 Vgl. H.F. Pitkin, The concept of representation, Berkeley/Los Angeles 1967, S. 209 f. Die nachstehenden Ausführungen zeigen auch, wie gut man daran tut, Repräsentation nicht als Individual-, sondern als Systemeigenschaft aufzufassen: „Repräsentativ“ ist allenfalls eine Vertretungskörperschaft, nicht aber ein einzelner Parlamentarier. 
der Verwirklichung kurzfristiger, mittelfristiger und langfristiger Interessen $\mathrm{zu}$ steuern. Doch keineswegs dürfen die Repräsentanten entlang der Überzeugung handeln, sie wüssten von sich aus oder aufgrund eines ,langen Planungsvorlaufs“" schon über das alles Bescheid und bräuchten auf weitere Meinungs- und Willensbekundungen der Repräsentierten deshalb nicht mehr zu achten.

Wenig überzeugend ist beim Blick auf Responsivität als Angelpunkt von Repräsentation der Standpunkt, eine Entscheidung sei nun einmal korrekt getroffen und müsse der Rechtsstaatlichkeit wegen jetzt eben vollzogen werden. Selbst in diesem Fall bleibt nämlich zu erläutern, ob derlei wirklich im Interesse der Bevölkerung liegt, und ist zu begründen, warum man eine Rechtslage eigentlich nicht so verändern dürfe, dass sie sich anschließend mit wichtigen Interessen der Repräsentierten deckt. Das gilt gerade dann, wenn die Bürgerschaft einen Entscheidungsprozess sozusagen verschlafen hat und sich nun zwar spät, doch immerhin artikuliert. Das Volk hat doch gerade in einem Repräsentativsystem viel geringere Aufmerksamkeits- und Artikulationsschulden als seine politische Klasse und deshalb jedes Recht, als „Prinzipal“ selbst dann noch gehört zu werden, wenn ihre ,Agenten“ zum nächsten Punkt auf der Agenda übergehen wollen. Allerdings meint Responsivität nur, dass die Repräsentanten sich auf die Interessenbekundungen der Repräsentierten ernsthaft und argumentierend einlassen; keineswegs wird verlangt, dass sie jenen Interessenbekundungen allein schon deshalb nachgeben, weil derlei von vielen Repräsentierten gewünscht wird.

Vielmehr muss für die Durchsetzung gewünschten Nachgebens der Repräsentanten seitens der Repräsentierten schon einige Mühe verlangt werden, damit nicht wirkungsvolle Willensbekundungen einiger weniger in die fallweise Herrschaft einer Minderheit über die Mehrheit münden. Die Artikulation von Opposition gegen rechtskräftige Beschlüsse der Repräsentanten muss aber schon auch ein erreichbares, klar definiertes Ziel haben und darf nicht aufs Hineinwirken ins politisch Diffuse beschränkt sein. Ein solches Ziel ist offenbar der Einsatz eines plebiszitären Instruments zur Korrektur einer bereits getroffenen Entscheidung. Sind solche Instrumente erst einmal politisch-praktisch - und nicht bloß rein rechtlich - verfügbar, so werden sie aller Erfahrung nach erhebliche Vorauswirkung entfalten: Weiß man nämlich um ihren sicher drohenden Einsatz, halten sie die politische Klasse von vornherein zu größerer als andernfalls zu erwartender Responsivität an. Das entlastet dann auch Wahlen von jener Funktionalisierung als Plebisziten über zentrale Wahlkampfthemen, die ihnen solange widerfährt, wie Wahlen für die Repräsentierten das Generalinstrument zur Erzwingung von Responsivität sind.

Am zweiten Eck des „Repräsentationsdreiecks“ findet sich: Repräsentanten und Repräsentierte vermögen unabhängig voneinander $\mathrm{zu}$ handeln, so dass es 
jederzeit zu Konflikten zwischen ihnen kommen kann. Was hieraus entsteht, kann man das „repräsentationskonstitutive Konfliktpotential“ nennen. Natürlich läge keine Repräsentationsbeziehung vor, sondern eine reine Herrschaftsbeziehung, wenn die politische Selbstartikulation einer Gesellschaft institutionell lahmgelegt wäre und die Repräsentanten unter Verweis auf - von ihnen vorgeblich erkannte - Interessen der Repräsentierten einfach das tun oder lassen können, was nur sie selbst für richtig halten. Daran identifiziert man typischerweise autoritäre oder totalitäre Herrschaft. Selbst in repräsentativen Demokratien schlägt aber das Pendel zu einer macht- und nicht zustimmungsgestützten Herrschaftsbeziehung dann aus, wenn sich die politische Klasse daran gewöhnen kann, unter Verweis auf Verfahren, die ihrer Prärogative vorbehalten oder inzwischen abgeschlossen sind, die Durchsetzung ihrer Entscheidungen gerade dann für faktisch oder rechtsstaatlich ,alternativlos“ zu erklären, wenn ein ins Gewicht fallender Teil der Repräsentierten nun einmal eine Alternative zur getroffenen Entscheidung wünscht.

Um einer solchen Entwicklung zu wehren, müssen die Repräsentierten zwischen den Wahlen über sämtliche Rechte pluralistischer Konfliktaustragung verfügen, nämlich von der Kommunikationsfreiheit über die Versammlungs- und Koalitionsfreiheit bis zum Demonstrationsrecht. Diese Möglichkeiten lassen sich nun aber auch um die unten erörterten plebiszitären Instrumente wie Volksantrag, Vorlageinitiative, Volksgesetzgebung und fakultatives Gesetzesreferendum ergänzen. Mit alledem kann man die Repräsentanten dann sogar punktgenau zur erwünschten Responsivität anhalten, indem man nämlich einen pluralistischen Konflikt bis hin zu dessen Entscheidung durch Plebiszit anfacht und zugleich dafür sorgt, dass die Volksvertreter durch Passivität das Risiko einer eigenen Niederlage nur steigerten. Taktisch höchst wichtige Vorauswirkungen bzw. „Antizipationsschleifen“ entstehen zumal dann, wenn am Ende des Konflikts anders als in rein repräsentativen Demokratien - nicht nur eine Entscheidung oder Nichtentscheidung des Parlaments stehen kann, sondern auch eine den parlamentarischen Mehrheitswillen womöglich überlagernde Entscheidung der Bürgerschaft. Aus der Faustregel, dass Repräsentation nur dann verlässlich entstehen kann, wenn das gesellschaftliche und politische Konfliktpotential institutionell und auf Dauer maximiert ist, lässt sich somit ableiten, dass in der Hand der Repräsentierten liegende plebiszitäre Instrumente gerade eine Repräsentationsbeziehung viel dichter machen, als sie es im Durchschnitt ohne diese Instrumente wäre.

Freilich bestünde eine Repräsentationsbeziehung auch dann nicht, wenn die Repräsentanten angesichts gesellschaftlicher Selbstartikulation einfach klein beigeben müssten und nur nachzuvollziehen hätten, was - wie im Fall von ,impera- 
tiven Mandaten“ - von anderen für sie (vor-) entschieden wurde. Im Grunde könnte man dann auf Repräsentation auch ganz verzichten und - gestützt auf die freilich sehr manipulationsträchtigen und den Kriterien demokratischer Transparenz schwerlich entsprechenden Möglichkeiten des Internet - zu einem System reiner Volksgesetzgebung oder zu einer völlig auf Referenden aufgebauten Demokratie übergehen. Wünscht man hingegen als wesentliche Dienstleistung der Repräsentanten beizubehalten, dass sie den empirisch vorfindbaren Volkswillen „veredeln“ und gerade die komplexen bzw. riskanten politischen Entscheidungen als erste vorbereiten und treffen, dann ergibt sich hieraus: Die Repräsentanten müssen das Recht haben, sich auch gegen - sogar heftig bekundete - Wünsche der Repräsentierten zu stellen, falls sie nämlich zur Ansicht gelangen, mitteloder langfristig wahrten ihre eigenen Urteile und Gestaltungsabsichten die Interessen der Repräsentierten oder das Gemeinwohl viel besser. Eben dafür benötigen sie aber ein freies Mandat, das ihnen auch nicht ohne eine erhebliche politische Anstrengung ihrer Prinzipale entzogen werden kann. Die Freiheit des Mandats darf allerdings nicht so weit gehen, dass die Repräsentanten um keinerlei Folgen für die Nutzung dieser Freiheit fürchten müssten. Vielmehr wirkt sich das „freie“ Mandat nur dann für die Repräsentierten nützlich aus, wenn die Repräsentanten grundsätzlich riskieren, für die Nutzung ihres Entscheidungsspielraums anschließend womöglich bestraft zu werden, etwa durch Niederlage bei einer Volksabstimmung, die sich gegen eine Parlamentsentscheidung richtet, oder durch Abwahl, wenn Abgeordnete nämlich die Repräsentierten gerade nicht davon überzeugen können, im Konfliktfall zwar gegen deren Wünsche, letztlich aber in deren Interesse gehandelt zu haben. ${ }^{22}$

Allerdings entfiele die besondere Dienstleistung von eigenverantwortlich agierenden Abgeordneten auch dann, wenn Repräsentanten die Verantwortung für eigenes Entscheiden ganz nach eigenem Ermessen im Weg eines Referendums an das Volk delegieren könnten. Dazu verhülfe ihnen das Recht, durch Parlamentsbeschluss, auf dem Umweg über die parlamentsgetragene Regierung oder mittels eines politisch funktionalisierten Staatsoberhaupts einfach dem Volk sol-

22 Damit ist offenbar das zentrale Anliegen des ,,imperativen“ Mandats erreicht: Repräsentanten sollen die selbstbekundeten Interessen der Repräsentierten niemals ignorieren können. Und somit ist die Leitidee des ,,imperativen Mandats“ auch dialektisch aufgehoben, nämlich innerhalb eines durch den ,Wiederwahlmechanismus“ mit demokratischer Willensbildung rückgekoppelten, vielleicht auch noch durch vom Volk in die Hand genommene plebiszitäre Instrumente vorauswirkend angeleiteten und eben dadurch praktisch eingegrenzten ,freien Mandats“. Vom Begreifen dieser Dialektik hängt ab, welche plebiszitären Instrumente man für einer repräsentativen Demokratie dienlich, welche anderen aber als ihr schädlich ansieht. 
che Entscheidungen vorzulegen, für die man selbst die politische Verantwortung scheut. Will man also Repräsentation, dann muss man auch und gerade bei einer Verbindung von Demokratie und Repräsentation den Repräsentanten die Möglichkeit nehmen, politischen Konflikten durch ihrerseits initiierte Referenden auszuweichen. Diese Einsicht kann man auf die Formel bringen: Plebiszitäre Instrumente in der Hand von Repräsentanten sind schlecht, weil sie Verantwortung für Entscheidungen von oben nach unten delegieren und letztlich zum Versickern bringen; hingegen sind plebiszitäre Instrumente in der Hand der Repräsentierten gut, weil sie die Repräsentanten zur politisch sanktionierbaren Parteinahme in repräsentationskonstitutiven Konflikten zwingen.

Das dritte Eck des „Repräsentationsdreiecks“ trägt gerade deshalb die Aufschrift: Es gelingt den Repräsentanten, das repräsentationskonstitutive Konfliktpotential im Großen und Ganzen befriedet zu halten. Hier lautet die Faustregel: Repräsentation liegt vor, wenn - bei Bestehen eines maximalen Konfliktpotentials - zwischen Repräsentanten und Repräsentierten häufige Konflikte nicht allzu heftig, heftige Konflikte aber nicht allzu häufig sind. $\mathrm{Zu}$ diesem Zustand führt zweierlei. Einesteils ist das praktizierte und glaubhaft gemachte Responsivität der Repräsentanten, andernteils problemlösende politische Führung, die wirksames Entscheidungshandeln gekonnt mit dessen Darstellung verbindet. Tatsächlich haben es nicht die Repräsentanten allein in der Hand, ob das für Repräsentation konstitutive Konfliktpotential wirklich befriedet ist; sie können bloß dafür sorgen, dass weder ihre Handlungen noch ihre öffentlichen Aussagen sonderliche Ansatzpunkte für durchschlagsfähige Kritik bieten. Eben das aber setzt die Repräsentanten unter jenen Leistungsdruck, der dem politischen System insgesamt zugute kommt.

Mitglieder der politischen Klasse werden nämlich dann in Schwierigkeiten geraten, wenn vom Volk beachtete Probleme von ihnen liegengelassen oder unzureichend angegangen werden. Also werden sie sich anstrengen, und zumal dann, wenn bei mangelhafter Responsivität der Einsatz plebiszitärer Instrumente droht. Und Repräsentanten werden wirklich in Bedrängnis geraten, wenn sie der Bevölkerung im Konfliktfall schlicht mit dem Hinweis kommen, jahrelang sei doch in partizipationsoffenen und rechtsstaatlich einwandfreien Verfahren auf die jetzt eben umzusetzende Entscheidung hingearbeitet worden. Gerade dann lässt sich nämlich das repräsentationskonstitutive Konfliktpotential nicht anhand einer „Legitimation durch Verfahren“ befrieden, sondern allein mittels „Legitimation 
durch Kommunikation““. ${ }^{23}$ Die letztere wird allerdings im Konfliktfall nicht in ihrer Ausprägung als politische Werbung akzeptiert, sondern nur als redlicher Austausch über Fakten, Perspektiven und Prioritäten. Nötig ist derlei erst recht, wenn ein auffallend großer, gebildeter und moderne Kommunikationstechnik nutzender Teil der Bevölkerung ein politisches Einzelvorhaben verhindern, durchaus aber nicht die freiheitliche demokratische Grundordnung antasten will. Dann steigern zusätzlich verfügbare plebiszitäre Instrumente die zu bewältigenden Kommunikationsherausforderungen gewiss.

Gerade einer gebildeten und partizipationswilligen Bürgerschaft die von ihr eingeforderte Kommunikation seitens der politischen Klasse zu verweigern, hat umso weniger Sinn, als es in einer repräsentativen Demokratie doch wesentlich auch darauf ankommt, dass sich die Bevölkerung repräsentiert empfindet. Weil aber die Vorstellungen über die Wirklichkeit und die Wirklichkeit selbst recht verschieden sein können, ${ }^{24}$ reicht es zur Sicherung demokratischer Legitimität nicht aus, dass Repräsentation in der beschriebenen Weise besteht. Vielmehr muss das Bestehen und Funktionieren demokratischer Repräsentation den Repräsentierten schon auch demonstriert und zum Gegenstand populären Vertrauens gemacht werden. Das aber gelingt - über einen guten Stand politischer Bildung hinaus sowie im konkreten Einzelfall - nur durch Kommunikation zur Sache, fallübergreifend auch durch Maßnahmen der symbolischen Selbstdarstellung von Repräsentationsinstitutionen und Repräsentanten, sowie - in hitzigen Konflikten - obendrein durch wirklich nutzbare plebiszitäre Instrumente, wie sie nachfolgend beschrieben werden. Diese stellen nämlich einesteils am Ende eines Konflikts sicher, dass die Repräsentanten den Wünschen der Repräsentierten folgen; und andernteils führen sie - nach Gewöhnung an ihre routinemäßige Verwendung - den Repräsentierten vor Augen, dass Repräsentanten unter demokratischen Bedingungen gar nicht anders können, als sie im oben umrissenen Sinn zu repräsentieren. Ist aber das erst einmal verstanden, dann verfängt es immer weniger, repräsentative Demokratie gegen ,direkte“ Demokratie auszuspielen.

Wider manches Erwarten können plebiszitäre Instrumente also wenigstens im Prinzip - und bei vernünftiger Ausgestaltung dann auch in der Praxis - sehr hilfreich sein, hinter der äußeren Form von Repräsentation diese selbst lebendig zu erhalten. Natürlich steigen die Ansprüche an die Repräsentanten, wenn die Repräsentierten punktuell durch Volksanträge und Vorlageninitiativen

23 Siehe N. Luhmann, Legitimation durch Verfahren (1969), 3. Aufl. 1993, und H. Oberreuter, Legitimation durch Kommunikation, in: J.W. Falter u.a. (Hrsg.), Politische Willensbildung und Interessenvermittlung, 1984, S. 238-253.

24 Zum hier einschlägigen Thomas-Theorem siehe Patzelt, Einführung (Fn. 6), S. 42. 
Responsivität erzwingen können und wenn sich das repräsentationskonstitutive Konfliktpotential durch Volksgesetzgebung und fakultative Gesetzesreferenden ausreizen lässt. Solchen größeren Herausforderungen dürften Politiker mit Schönwetterkarrieren ebensowenig gerecht werden können wie jene, deren Virtuosität sich auf die innerparteiliche Absicherung beschränkt, und zwar ohne von ebenso großer Fähigkeit beim Umgang mit teils aufgebrachten Bürgern, teils abgebrühten und finanzstarken Protestexperten flankiert zu werden. Solch veränderter Selektionsdruck dürfte der Zusammensetzung unserer politischen Klasse aber dahingehend gut tun, dass sie sich besser als bislang auch bei innenpolitisch erschwerten Führungsaufgaben bewähren muss. Gewöhnt sich dann auch noch die Bürgerschaft durch wiederholte Nutzung plebiszitärer Instrumente daran, dass diese nicht Spielzeuge zum Abreagieren von Politikerverdrossenheit sind, sondern mit ihnen folgenreiche Entscheidungen getroffen werden, dann mag vielleicht auch politische Bildung sich hin zu einem Niveau und einer Breite entwickeln, wie sie einem demokratischen Gemeinweisen gut tun. Nur brauchen auch diese Früchte Zeit zum Wachsen und günstige Umstände zum Reifen.

\section{Rahmenbedingungen für nützliche plebiszitäre Instrumente}

Welche plebiszitären Instrumente wären also wünschenswert, wenn repräsentative Demokratie nicht beeinträchtigt, sondern verbessert werden soll? Als deren zentrale Herausforderung hat sich erwiesen, für größtmögliche Responsivität der Repräsentanten unter Beibehaltung von deren Erstentscheidungspflicht zu sorgen. Dafür sind Volksanträge und Vorlageninitiativen, sind Volksgesetzgebung und fakultative Gesetzesreferenden geeignet, womöglich auch plebiszitär herbeizuführende Parlamentsauflösungen, und in einigen Fällen vielleicht obendrein obligatorische Referenden zu grundlegend wichtigen Entscheidungsgegenständen. ${ }^{25}$ Doch vorab verdienen einige grundsätzliche Überlegungen gleichsam ,,vor die Klammer" gezogen zu werden, damit sie nicht bei der Vorstellung jedes einzelnen Instruments aufs Neue zu erwähnen sind. ${ }^{26}$

25 Die Bezeichnungen für die genannten plebiszitären Instrumente variieren in der politischen Sprache. Weil im Folgenden aber stets klar wird, was jeweils gemeint ist, kann auf größeren definitorischen Aufwand verzichtet werden.

26 Für alle rechtlichen Erwägungen bei der Nutzung plebiszitärer Instrumente, zu deren Verfügbarkeit und Nutzung in Deutschland sowie ganz allgemein zu diesem Thema siehe $P$. Neumann, Sachunmittelbare Demokratie im Bundes- und Landesverfassungsrecht unter besonderer Berücksichtigung der neuen Länder, 2009. 
Für alle plebiszitären Instrumente müssen Antrags-, Beteiligungs- und Zustimmungsquoren festgelegt werden. Ein Antragsquorum - ausgedrückt in Prozent der Wahl- bzw. Abstimmungsberechtigten oder in einer absoluten Stimmenzahl - legt fest, wie viel Unterstützung in Form von Unterschriften vor$a b$ mobilisiert werden muss, um ein plebiszitäres Instrument überhaupt zum Einsatz zu bringen. Deshalb wird es oft auch „Unterstützungsquorum“ genannt. Faktisch geht es hier um einen Test darauf, ein wie großer Teil der Bevölkerung tatsächlich hinter einem Volksantrag oder dem Wunsch nach einem fakultativen Gesetzesreferendum steht. Also haben Antragsquoren eine Filterfunktion: Sie sollen einerseits sicherstellen, dass auch eine Minderheit der Bürgerschaft für den politischen Prozess folgenreich ihre Stimme erheben kann, und andererseits sollen sie verhindern, dass es zu einer Vielzahl von alsbald unüberschaubaren und die Wählerschaft nicht mobilisierenden Einzelaktionen ohne sonderliche Wirkung kommt. Folglich muss eine nur mit Anstrengung zu nehmende Hürde aufgebaut werden.

Es wird weise sein, die Höhe dieser Hürde nicht willkürlich, sondern nach inhaltlichen Erwägungen festzulegen. ${ }^{27}$ Diese beginnen mit der Beobachtung, dass sich allenfalls 50 Prozent der Bevölkerung für Politik interessieren. Auf diesen Wert scheint denn auch die Wahlbeteiligung zumindest bei Landtags- und Europawahlen zu sinken. Bei Volks- sowie Bürgerentscheiden in Deutschland rangiert die Beteiligung zwischen ungefähr 25 und 70 Prozent, bei Volksentscheiden und Referenden in der Schweiz zwischen 27 und 79 Prozent, wobei die durchschnittliche Abstimmungsbeteiligung dort bei etwa 40 Prozent liegt. ${ }^{28} \mathrm{Im}$ Übrigen sind in Deutschland keine drei Prozent der Wahlberechtigten Mitglied und dann meist inaktiv - einer Partei. Die Mitgliedschaft in Verbänden und Vereinen des ,,vorpolitischen Raums“ liegt hierzulande zwar viel höher, erreicht die höchsten Werte aber dort, wo solche Organisationen - etwa Sportvereine - gera-

27 Für Bürgerentscheide auf Gemeindeebene liegen in den deutschen Bundesländern die Antragsquoren zwischen 2\% (Hamburg, auf Bezirksebene) und 17\% (Obergrenze in Thüringen), auf Kreisebene zwischen 0\% (Baden-Württemberg, Berlin auf Bezirksebene, Stadt Bremen, Stadt Bremerhaven, Hamburg auf Bezirksebene, Hessen, Thüringen) und $15 \%$ (Sachsen, Obergrenze in Thüringen). Für Volksbegehren auf Landesebene liegen die Antragsquoren zwischen derzeit rund $3,8 \%$ der Wahlberechtigten (Brandenburg, wo 80.000 Unterschriften gefordert sind) und 20\% (Hessen, Saarland; ferner in Berlin und Bremen für verfassungsändernde Entwürfe). Das alles weist auf wenig Systematik und viel politischen Dezisionismus hin.

28 Alle Zahlen nach Neumann, Sachunmittelbare Demokratie (Fn. 26), S. 387. 
de nicht mit auch politischem Engagement verbunden sind. Gewiss wird man Antragsquoren deutlich oberhalb des Prozentsatzes der Parteimitglieder festlegen müssen, wenn die Unterstützung von mehr Bürgern als nur den ohnehin politisch aktiven für ihren Einsatz erforderlich sein soll. Will man plebiszitäre Instrumente aber wirklich nutzbar machen, darf das Antragsquorum nicht abschreckend hoch sein. Falls nun der zentrale Mehrwert plebiszitärer Instrumente für eine repräsentative Demokratie darin liegt, deren Responsivität zu steigern, kann die FünfProzent-Hürde für den Einzug einer Partei ins Parlament ein plausibles Antragsquorum markieren: Bislang schon braucht es diesen Anteil der Wahlberechtigten, um die politische Klasse zu zwingen, sich auf die Themen oder Anliegen einer (neuen) Partei einzulassen.

Zwar liegen derzeitige Antragsquoren deutlich unter fünf Prozent der Wahloder Abstimmungsberechtigten. Sie zu überwinden erschließt bislang aber nur begrenzte plebiszitäre Möglichkeiten und führt meist an die zwar inzwischen sinkenden, durchaus aber noch hohen, nicht selten auch hinter Zustimmungsquoren versteckten Hürden hoher Beteiligungsquoren. Für wenig Aufwand bekommt man derzeit also auch nur wenig wirklichen Einfluss. Wenn aber - wie im Folgenden angeregt - die Beteiligungsquoren abgeschafft würden, dann wäre es nur konsequent, den Preis für solchen Zugewinn an Einfluss moderat heraufzusetzen. Plausiblerweise sollte das Antragsquorum nämlich um so höher liegen, je mehr vor allem ihm die Filterfunktion für die tatsächliche Nutzung eines plebiszitären Instruments zukommt. Außerdem sollte ein Antragsquorum wohl um so höher liegen, je formloser sich die für seine Erreichung erforderlichen Unterschriften sammeln lassen, und um so niedriger, je mehr zeitlicher Aufwand hinter jeder einzelnen Unterschrift stehen muss. Am niedrigsten muss es sein, falls für die unterstützende Unterschrift eine Behörde aufzusuchen ist. Im Übrigen muss bei Antragsquoren durch geeignete Ausführungsvorschriften Manipulation bei der Unterschriftensammlung möglichst unterbunden sowie dafür gesorgt werden, dass die Frist zum Aufbringen der Unterschriften nicht abschreckend kurz ist.

Ein Beteiligungsquorum legt fest, ein wie großer Teil der Wählerschaft sich an einer Volksabstimmung beteiligen muss, damit ihr Ergebnis auch verbindlich wird. Auf den ersten Blick scheint es so zu sein, als müsse das Beteiligungsquorum über dem Antragsquorum liegen: Immerhin geht es hier nicht nur um die Einleitung eines plebiszitären Verfahrens, sondern um eine das Staatsvolk bindende Entscheidung. Ein Quorum von 50 Prozent der Wahl- bzw. Abstimmungsberechtigten bzw. entsprechend der durchschnittlichen Wahlbeteiligung im letzten Jahrzehnt wirkt dann plausibel. Doch auf den zweiten Blick erkennt man, dass Beteiligungsquoren nur zu taktischen Verhaltensweisen einladen, die 
dem Zweck plebiszitärer Instrumente einfach zuwiderlaufen. Wer nämlich eine plebiszitär gewünschte Entscheidung verhindern will, muss sich bei einem hohen Beteiligungsquorum gar nicht erst auf eine Auseinandersetzung in der Sache einlassen, sondern kann durch Aufruf zum Abstimmungsboykott oder schlicht durch Nichtthematisierung des Streitgegenstands gleichsam „Wahlkampf gegen die Abstimmungsbeteiligung“ machen. Ein solches taktisches Verhalten beraubt aber das ganze plebiszitäre Verfahren seines Sinns, die politische Klasse zur Responsivität anzuhalten. Also ist ein hohes Beteiligungsquorum gerade nicht angebracht.

Die angemessene Höhe des Beteiligungsquorums ergibt sich wohl aus einer der beiden nachfolgenden Überlegungen. Einerseits ist es plausibel, dass Antrags- und Beteiligungsquorum gleich hoch sein sollten: Wenn sich nämlich weniger Bürger an einer Volksabstimmung beteiligen, als einst ihr Vorhaben auf den Weg gebracht haben, dann kann man vermuten, das einst beförderte Anliegen habe seine politische Bedeutung inzwischen verloren. Andernteils ist es auch plausibel, überhaupt kein Beteiligungsquorum anzusetzen: Dann entfällt von vornherein die Möglichkeit eines „Wahlkampfs gegen die Abstimmungsbeteiligung“ und wird es für die Repräsentanten hochriskant, die Inhalte des plebiszitären Unterfangens zu ignorieren. Ganz im Gegenteil müssen sie stark in kommunikative Führung investieren, wenn sie nicht von einer faktischen Minderheit der Bevölkerung überspielt werden wollen. Eben das aber verbesserte - aufgrund der oben dargestellten Wirkungszusammenhänge - die Repräsentationsleistung des Gesamtsystems. Die ohne Beteiligungsquorum grundsätzlich gegebene Möglichkeit einer aktiven Minderheit, sich gegen eine apathische oder träge Mehrheit durchzusetzen, wird nämlich spätestens dann massive Vorauswirkungen auslösen, wenn ein erstes Mal eine Volksabstimmung zu einem mehrheitlich gerade nicht gewünschten, durch breite Apathie aber ermöglichten Ergebnis führte. Politischer Erfahrung nach kann man deshalb auf die alsbaldige Entstehung von „verbesserter Repräsentation dank Vorauswirkung plebiszitärer Instrumente“ vertrauen. Im Übrigen kennt man in vielen Staaten auch bei Wahlen keine Mindestwahlbeteiligung. Deren Fehlen wird auch nur selten bedauert. Viel öfter beklagt man dort, wo es auch für Wahlen Beteiligungsquoren gibt, deren politikhemmende Wirkung. Es ist also abstimmungstheoretisch eher die Einführung eines Beteiligungsquorums rechtfertigungsbedürftig als der Verzicht darauf. Im Übrigen scheint es klüger zu sein, alle Quoren in Prozent der jeweils Wahlberechtigten auszudrücken und als in absoluten Unterschriften- oder Stimmenzahlen, weil nur dies verzerrungslos dynamischem Bevölkerungswandel gerecht wird. 
Ein Zustimmungsquorum legt fest, ein wie großer Anteil der Wählerschaft eine plebiszitäre Abstimmungsfrage bejaht haben muss, damit diese Antwort verbindlich wird. Weil also ein Zustimmungsquorum selbst dann eine wichtige Filterwirkung hat, wenn vorab auf ein Beteiligungsquorum verzichtet wurde, lässt sich plebiszitären Instrumenten durch hohe Zustimmungsquoren - die auch ihrerseits notwendigerweise als ein (weiteres) Beteiligungsquorum wirken - von vornherein ihr Nutzen entziehen. ${ }^{29}$ Wenn man das gerade nicht will, sollte man besser auf besondere Zustimmungsquoren verzichten und - wie meist bei parlamentarischen Entscheidungen - die einfache Mehrheit der Stimmen genügen lassen. Bei für besonders wichtig gehaltenen Abstimmungen kennen allerdings auch Parlamente Zustimmungsquoren. Also spricht nichts dagegen, sie grundsätzlich für plebiszitäre Instrumente ebenfalls zu erwägen und für die besonders folgenreichen unter ihnen auch vorzusehen, etwa für plebiszitär bewirkte Parlamentsauflösungen. Allerdings sollte die Höhe eines Zustimmungsquorums ebenfalls nicht ohne inhaltliche Begründung festgelegt werden. Recht plausibel wäre es, das Zustimmungsquorum ans Antragsquorum zu binden: Wenn bei der Volksabstimmung nicht mindestens derselbe Prozentsatz der Wahlberechtigten wie jener, der einst die Nutzung eines plebiszitären Instruments ins Werk setzte, weiterhin das damals plebiszitär begehrte Ziel erreichen will, dann ist aus einer Minderheitsbewegung offensichtlich gerade keine Mehrheitsströmung geworden; und folglich ist es dann auch nicht plausibel, dass diese stagnierende Minderheit nun der faktisch schweigenden Mehrheit ihren Willen sollte aufzwingen können.

29 Für Bürgerentscheide auf Gemeindeebene liegen in den deutschen Bundesländern die stets auch ein Beteiligungsquorum implizierenden - Zustimmungsquoren zwischen $0 \%$ (Hamburg, auf Bezirksebene, wo - wie im Folgenden empfohlen - die einfache Mehrheit der Abstimmenden in jedem Fall als ausreichende Zustimmung gewertet wird) und 30\% (Stadt Bremerhaven, Rheinland-Pfalz, Saarland). Auf Kreisebene liegen sie zwischen recht nahe 0\% (in Baden-Württemberg, Berlin auf Bezirksebene, Stadt Bremen, Stadt Bremerhaven, Hamburg auf Bezirksebene, Hessen, Thüringen) und 30\% (RheinlandPfalz, Saarland). Auf Landesebene schwanken in Deutschland die Zustimmungsquoren bei einfachen Gesetzen zwischen 0\% (Bayern, Hessen, Sachsen) und einem Drittel der Abstimmungsberechtigten (Baden-Württemberg, Mecklenburg-Vorpommern). Die Zustimmungsquoren für Verfassungsänderungen liegen regelmäßig höher. Bayern mit $25 \%$ ist hier das Schlusslicht; Thüringen hat ein Zustimmungsquorum von $40 \%$. In den anderen Bundesländern liegt es bei $50 \%$, wobei bisweilen außerdem noch eine Zweidrittelmehrheit unter den Abstimmenden verlangt wird. Alles in allem zeigt sich auch hier ein recht willkürliches Muster. 
In einem föderalen Staatswesen wie dem unseren muss bei bundesweiten Volksabstimmungen dem Grundsatz der Mitwirkung der Länder an der Gesetzgebung des Bundes Rechnung getragen werden. Dies lässt sich nach dem Schweizer Modell dahingehend bewerkstelligen, dass es bei Volksabstimmungen, ja womöglich auch schon beim Erreichen des Antragsquorums, nicht nur auf das „Volksmehr“ bzw. „Stimmenmehr“ ankommt, sondern auch auf das „Ständemehr“. Das bedeutete im deutschen Fall, dass nicht nur insgesamt, sondern auch in einer Mehrheit der Bundesländer die Volksabstimmung gewonnen oder das Antragsquorum überwunden werden muss. Diese Regel sorgte ferner dafür, dass nicht die Abstimmenden in den bevölkerungsstarken Ländern umstandslos die Abstimmenden in den bevölkerungsarmen Ländern majorisieren könnten, und sich bei nationalen Referenden nicht umgekehrt einfach regionale Interessen durchsetzten. Zwar können mehrere kleinere Länder immer noch die größeren Länder blockieren. Doch das ist immer schon im Bundesrat sowie im Rat der EU der Fall und wird dort weniger als Problem denn als Form des Minderheitenschutzes verstanden.

Einmal eingeführt, würde dieses Erfordernis einer „doppelten Mehrheit“ nämlich sowohl der Abstimmenden als auch der Länder, in denen die jeweilige Volksabstimmung gewonnen wird - unter den Initiatoren eines plebiszitären Instruments genau jene Ausgleichs- und Aushandlungsprozesse herbeiführen, die auch der parlamentarischen Mehrheitsbildung vorauszugehen pflegen. Natürlich lässt sich die „Regel der doppelten Mehrheit“ auch dahingehend modifizieren, dass das Ländermehr den für ein inhaltlich äquivalentes Gesetz geltenden Mehrheitsverhältnissen im Bundesrat entsprechen muss, oder dass eine „Mehrheit unter den Ländern“ nur dann festgestellt werden kann, wenn in den Ländern mit „erfolgreicher Volksabstimmung“ auch die Mehrheit der Bevölkerung ihren Erstwohnsitz hat. Umgekehrt könnte bei plebiszitären Instrumenten, welche Gesetze betreffen, die der Zustimmung des Bundesrates nicht bedürfen, auf das Erfordernis einer Ländermehrheit bei der Volksabstimmung verzichtet werden. Insgesamt scheint es nur einiger staatsrechtlicher Findigkeit zu bedürfen, um plebiszitäre Instrumente mit dem bundesdeutschen Gesetzgebungsprozess kompatibel zu machen. Für dessen demokratische Legitimierung aber erlaubt die Verfassung doch jetzt schon, nicht nur an Wahlen, sondern auch an Volksabstimmungen zu denken. 
III. Bindewirkung und Korrekturen plebiszitärer Entscheidungen

Gewiss ist der Einsatz plebiszitärer Instrumente sinnvoll nur dann, wenn die Ergebnisse ihrer Nutzung auch Bindewirkung entfalten. Dem widerspräche völlig die Möglichkeit eines Parlaments, das der politischen Klasse unwillkommene Ergebnis einer Volksabstimmung gleich wieder durch einen Gesetzesbeschluss zu „korrigieren“. Da freilich auch der parlamentarische Gesetzgeber die Möglichkeit hat, sich zu korrigieren, wäre es unplausibel, wenn Ergebnisse von Volksabstimmungen jeglicher Korrekturmöglichkeit entzogen würden.

Zur Lösung dieses Problems lässt sich einesteils vorsehen, dass Volksabstimmungen auf dem gleichen Weg, wie sie einst zustande kamen, auch einem Korrekturverfahren unterzogen werden können: nämlich durch Überwindung eines entsprechenden Antragsquorums mit anschließender neuerlicher Volksabstimmung. Andernteils sollen im System repräsentativer Demokratie die Repräsentanten ja gerade nicht aus ihrer eigenen Verantwortung entlassen werden. Also ist es angemessen, dass auch Parlamente die Ergebnisse von Volksabstimmungen korrigieren können. Freilich darf das nicht ohne Vetomöglichkeiten eben jenes Volkes geschehen, das doch - und sei es auch ohne Beteiligungsquorum zuvor seinen jetzt mit Korrekturwünschen konfrontierten Willen durchgesetzt hat. $\mathrm{Zu}$ einem schlüssigen Verfahren führte einerseits die Regel, das jeweils nächste Parlament dürfe durch eigenen Gesetzesbeschluss das Ergebnis einer Volksabstimmung korrigieren. In diesem Fall kann man nämlich den Korrekturwunsch - und bestünde er auch einfach in der Aufhebung des vom Volk beschlossenen Gesetzes - zum Wahlkampfinhalt machen, der dann seinerseits für die Wahlentscheidung den Ausschlag geben mag. Andererseits kann man die Möglichkeit einer Selbstauflösung des Parlaments einführen, so dass sich sehr rasch auf dringliche Korrekturwünsche reagieren lässt. Im Übrigen spricht nichts dagegen, auch vom Volk beschlossene Gesetze den üblichen abstrakten und - im Wege der Richtervorlage - konkreten Normenkontrollverfahren zu unterziehen. Die Demokratie entfaltet sich schließlich im Rahmen des materiellen Rechtsstaates, steht aber nicht über ihm.

IV. Regelungsmaterien und Zulässigkeitskriterien

Zweifellos bringt der Einsatz plebiszitärer Instrumente kaum Gewinn für die repräsentative Demokratie, wenn ihr Einsatzbereich auf nur wenige und unwichtige Themen beschränkt wird. Zumal der Ausschluss von Entscheidungsfragen mit fiskalischen Folgewirkungen bringt plebiszitäre Instrumente um einen großen 
Teil ihrer möglichen Wirksamkeit. Oft wird zwar für den Ausschluss finanziell folgenreicher Themen mit dem Hinweis darauf argumentiert, andernfalls sei das Budgetrecht des Parlaments als Eckstein von dessen Machtstellung gefährdet. Dieses Argument verkennt aber einen wichtigen Funktionswandel des Parlaments gerade in Budgetfragen.

Als nämlich die Parlamente noch Ständeversammlungen waren und ihre Mitglieder die beschlossenen Steuern oder einmaligen Abgaben selbst bzw. im eigenen Herrschaftsbereich aufbringen mussten, wirkte das parlamentarische Budgetrecht recht leicht wie eine Ausgabenbremse. Seit aber die Parlamente aus demokratisch gewählten Abgeordneten bestehen, die einerseits aus ihrem eigenen Einkommen zum Staat nicht mehr beitragen als ihre steuerlich gleich veranlagten Mitbürger, die andererseits aber gerade durch Steigerung der öffentlichen Ausgaben - etwa für „mehr soziale Gerechtigkeit“, für „sicherere Arbeitsplätze“ oder für „,bessere Infrastruktur“ - ihre Wiederwahlchancen vergrößern können, entfiel diese im Eigeninteresse liegende Ausgabenbremse. Seither stiegen die Staatsausgaben immer mehr. Also wird - wenigstens in Deutschland - nun verfassungsrechtlich zu limitieren versucht, was sich aufgrund so drastisch veränderter Anreizstrukturen allein politisch gerade nicht mehr begrenzen lässt. In dieser Lage ist allenfalls noch einem Großteil der Bürgerschaft aus Eigeninteresse daran gelegen, die Staatsausgaben - und damit die Staatsschulden bzw. künftigen Zins- und Tilgungslasten - nicht weiter steigen zu lassen: jener Teil nämlich, der aus dem eigenen Einkommen für das alles aufzukommen hat.

Was also auf den ersten Blick wie eine Aushöhlung des Parlamentsrechts auf Kontrolle der Staatsausgaben und somit wie der Aufbau eines plebiszitären Selbstbedienungsladens aussieht, erweist sich auf den zweiten Blick als fallweise Zurückverlagerung des fiskalischen Entscheidungsrechts in die Hände derer, die den Staat finanziell tragen: nämlich der Bürger. Der Blick in Staaten wie die Schweiz, wo Volksabstimmungen auch die Staatsfinanzen betreffen können, erweist außerdem, dass derlei wohl eher zur Begrenzung als zur Ausdehnung der Staatsausgaben führt. Im Übrigen lassen sich in die Gesetze zur Einführung plebiszitärer Instrumente auch Regelungen der Art aufnehmen, dass jedes plebiszitäre Verlangen nach einer konkreten Ausgabenerhöhung durch einen zusammen mit dessen Annahme verbindlich werdenden Einsparungsvorschlag gekoppelt werden muss, Kreditaufnahmen ohne Finanzierungsplanung also gerade nicht plebiszitär bewirkt werden dürfen.

Insgesamt wird die Regel sinnvoll sein, dass mit plebiszitären Instrumenten im räumlichen Zuständigkeitsbereich einer Vertretungskörperschaft alle jene Themen politisch bearbeitet werden dürfen, welche der Regelsetzungs- und Einzelfallentscheidungskompetenz dieser Vertretungskörperschaft anvertraut sind. 
Dann versteht sich aber auch von selbst, dass für die Inhalte und Grenzen von Volksentscheiden dieselben rechtlichen Rahmenbedingungen gelten müssen wie für Parlamente und Regierungen auch, nämlich vom Minderheitenschutz über das Rückwirkungsverbot und den Verhältnismäßigkeitsgrundsatz bis hin zu den Prinzipien des materiellen Rechtsstaates. Und nicht minder ist klar, dass zur Sicherung all dessen eine Zulässigkeitskontrolle der Entscheidungsvorlagen aller plebiszitären Instrumente vorzusehen ist. Ob diese Zulässigkeitskontrolle in der Hand ausgerechnet von Parlamentspräsidenten liegen sollte, bleibt zu diskutieren; Konsens hingegen dürfte sein, dass alle Zulässigkeitsentscheidungen auch binnen kurzer Frist gerichtlich überprüfbar sein müssen. Im Übrigen müssen Entscheidungsregeln für den Fall vorgesehen werden, dass im Mehr-EbenenSystem von kommunaler Selbstverwaltung, Landes- und Bundeskompetenzen plebiszitäre Instrumente zu widersprüchlichen Ergebnissen führen. Das wäre etwa dann der Fall, wenn auf kommunaler Ebene die Durchführung einer Baumaßnahme plebiszitär unterbunden würde, deren Rahmenplanung auf Landesebene plebiszitär beschlossen wurde. Will man in solchen Fällen nicht dem St. Florians-Prinzip Wirkungsräume eröffnen, wird man auch hier nach einer Hierarchie von Rechtsnormen verfahren müssen: So wie Landesgesetze kommunalen Vorschriften vorgehen, müssen Volksabstimmungen auf Landesebene solche auf kommunaler Ebene und solche auf Bundesebene jene auf Landesebene aufheben können. Schließlich ist ein Bundesland kein Bund unabhängiger Kommunen und Deutschland keine Föderation souveräner Staaten.

\section{Plebiszitäre Instrumente und der Bildungsstand der Bevölkerung}

Zwar ist der Einwand nicht von der Hand zu weisen, plebiszitäre Instrumente überforderten die Entscheidungsfähigkeit der Bürgerschaft, weil ein Großteil der Bürger weder die erforderlichen Informationen besäße noch, mit ihnen ausgestattet, sie angemessen verstehen könne. Tatsächlich hat man dieses Argument im 19. Jahrhundert schon gegen die Einführung des allgemeinen Wahlrechts in Anschlag gebracht. Niemand wird ferner bestreiten, dass viele Wahlentscheidungen uninformiert, rein nach Gefühl und von ihren Ergebnissen her niederschmetternd falsch getroffen werden. Dennoch zeigt der vergleichende Blick in die internationale Staatenpraxis, dass Länder mit allgemeinem Wahlrecht im Durchschnitt weniger nachteilige politische Weichenstellungen vornehmen als Staaten mit autokratischer Elitenherrschaft. Es scheint somit eher die durch allgemeine Wahlen ausgelöste breite Erörterung öffentlicher Angelegenheiten als die Rationalität jeder individuellen Wahlentscheidung zu sein, die einem auf freie Wahlen ge- 
gründeten Gemeinwesen Vorteile beschert. Das vor Augen, wird man es mit dem Argument nicht übertreiben dürfen, die Bürger seien zwar informiert und klug genug, Parteilisten und Einzelbewerber für öffentliche Ämter zu wählen, doch grundsätzlich kaum in der Lage, sich über einen Volksantrag oder einen Gesetzentwurf ein vernünftiges Urteil zu bilden. Umso weniger zieht dieses Argument dann, wenn es - wie bei den nachstehend beschriebenen plebiszitären Instrumenten - auch gar nicht darum geht, komplexe Sachverhalte entlang simpler ja/neinAlternativen zu entscheiden, und wenn ohnehin die politische Entscheidungsfindung nicht allein in die Hände des Volks gelegt werden soll.

Immerhin ist auch kein Parlamentarier kompetent auf allen Politikfeldern, über die er in Fraktion und Plenum entscheidet; er verlässt sich dort vielmehr auf seine spezialisierten Kollegen. Entsprechend hindert nichts daran, bei jeder Volksabstimmung - wie in der Schweiz - jedem Abstimmungsberechtigten zusammen mit seiner „Wahlbenachrichtigung“ auch ein amtliches „Abstimmungsheft" zukommen zu lassen, in dem alle erforderlichen Informationen und Sachargumente zusammengefasst sind, und zwar unter Verantwortung des jeweiligen Wahlleiters nach möglichst einvernehmlicher Redaktion aller Abstimmungskonkurrenten. Die Kosten dafür sind einfach „Kosten der Demokratie“, die sich immer wieder als höchst renditeträchtige Investitionen erweisen. $\mathrm{Zu}$ deren Rendite gehört, dass sich schon nach wenigen Jahren der politische Bildungsstand eines Bürgers, der jährlich an zwei, drei Volksabstimmungen teilgenommen und sich vorab anhand des Abstimmungsheftes sachkundig gemacht hat, dem eines politisch gleich interessierten Bürgers klar voraussein dürfte, der nur an Wahlen teilnehmen darf. Gewiss wird derlei den politisch besonders interessierten Teil der Bevölkerung viel weiter voranbringen als die politisch weniger Interessierten. Doch derlei ist eben Folge des Rechts freier Bürger auch auf politisches Desinteresse und kann somit nicht mit überzeugenden Argumenten gegen den Ausbau plebiszitärer Instrumente vorgebracht werden. Schon gar kein überzeugender Einwand gegen Volksabstimmungen ist der Hinweis, in deren Vorfeld könne es zu manipulativen Einflussnahmen der jeweiligen Befürworter oder Gegner auf die Abstimmungsberechtigten kommen. Denn zu alledem kommt es natürlich auch in Wahlkämpfen - und trotzdem regt kaum einer an, zur Verhinderung solcher Manipulationen einer derart wichtigen Entscheidung solle man besser auf Wahlen verzichten.

Außerdem ist aufgrund von Erfahrungen wie jenen mit dem Stuttgarter Bahnhofsumbau noch ein weiterer Wirkungszusammenhang in Rechnung zu stellen. Zumal komplexe Planungsentscheidungen werden nämlich seitens der politischen Klasse sowie der Verwaltungseliten ganz sach- und ordnungsgemäß in viele Teilentscheidungen aufgegliedert, und zwar besonders dann, wenn unter- 
schiedliche öffentliche Belange betroffen sind oder mehrere staatliche Ebenen zusammenwirken müssen. Für jeden Einzelschritt ergeben sich dann unterschiedliche Zuständigkeiten, gegebenenfalls auch Beteiligungsmöglichkeiten. Bis dann alle Einzelschritte getan sind, vielleicht auch immer wieder angehalten durch verwaltungsgerichtliche Verfahren, vergehen viele Jahre. Am Anfang des Planungs- und Entscheidungsprozesses hat dann zwar eine fraglos dazu legitimierte Vertretungskörperschaft eine Grundsatzentscheidung getroffen, womöglich auch in Übereinstimmung mit dem damals mehrheitlichen Bürgerwillen. Doch nach vielen Jahren sind nicht wenige der damaligen Entscheider gar nicht mehr im Amt und wurden zwischenzeitliche Entscheidungen oft nicht mehr im Blick auf die Gesamtplanung durchdacht, sondern vor allem unter dem Gesichtspunkt behandelt, nun endlich den nächsten Schritt voranzukommen. Was am Ende eines solchen komplexen Planungs- und Entscheidungsprozesses herauskommt, hat dann mitunter gar nicht mehr jemand als solches entschieden, sondern eine jeweils neue Gruppe von Amtsträgern einfach übernommen und weitergegeben wie ein sich nach seinen ganz eigenen Regeln entwickelndes Gewächs. Verantwortung gab es dann nur insofern, als man dieses Gewächs weiterreichte; doch die Verantwortung dafür, es insgesamt richtig $\mathrm{zu}$ beschneiden oder $\mathrm{zu}$ bepfropfen, ist einfach versickert. Die Bürgerschaft aber wird eines Tages mit einem Gesamtergebnis konfrontiert, über dessen im Lauf der Jahre entstandene Gesamtgestalt nie von einem zur Verantwortung zu ziehenden Politiker entschieden wurde. Also ist es höchst verständlich, wenn ein Teil der Bürger sich mit einem solchen Gesamtergebnis nicht umstandslos einverstanden erklärt oder wenn überhaupt die politische Basis eines solchen Großvorhabens im Lauf der Jahre geschwunden ist.

Befördert wird derlei leicht, wenn es über diese Jahre hinweg auch keine das Gesamtvorhaben und seinen Sinn vor Augen führenden Kommunikationsanstrengungen der politischen Klasse gegeben hat, also „Legitimation durch Kommunikation" unterblieben ist. Gewiss kann die Bürgerschaft - und sicher auch ein nennenswerter Teil der politischen Klasse - im Nachhinein dann nicht ohne größere Anstrengungen nachvollziehen, warum es mit dem jetzt nach überaus langer Planungszeit zur Verwirklichung anstehenden Vorhaben gerade so gekommen ist, wie es eben gekommen ist. Doch zweifellos sind die Bürger in der Lage, sich darüber ein Urteil zu bilden, ob ihnen das Gesamtergebnis gefällt und sie eine Verwirklichung des Großprojekts wünschen. Auch ist es nicht von vornherein aussichtlos, der Bürgerschaft die womöglich nachteiligen Folgen eines jetzt, nach Abschluss des Planungsverfahrens, vollzogenen Projektabbruchs vor Augen zu führen. Verlangt man also nur jenes Gesamturteil, über das hinaus man auch die meisten Repräsentanten bei größeren Projekten selten fordert, lässt 
sich kein überzeugender Grund finden, warum ein entsprechender Volksentscheid die Repräsentierten überfordern sollte. Hätten hingegen die Repräsentanten mit der Nutzung eines plebiszitären Instruments ,in letzter Minute“ ohnehin rechnen müssen, dann wären sie auch schwerlich so nachlässig bei der Kommunikation über das anstehende Großvorhaben gewesen. Insofern also Volksentscheide selbst zu komplexen Materien schon im Weg der Vorauswirkung eine viel breitere Erörterung öffentlicher Angelegenheiten zeitigen, als derlei ansonsten zu erwarten wäre, verfehlt der Verweis auf eine mögliche Überforderung der Bürger bei besonderen Entscheidungen genau den Kern der Sache: Es geht um die Einführung eines institutionellen Mechanismus, der die Repräsentanten dazu anhält, auch bei langjährigen und komplexen Entscheidungsprozessen die legitimierende Kommunikation mit der Bürgerschaft zu suchen. Im Übrigen stellt Demokratie ohnehin nicht ,richtige“ Entscheidungen in Aussicht, sondern nur solche, die eine recht große Chance auf repressionsfreie Akzeptanz haben.

\section{Der Mehrwert einer kontinuierlichen Nutzung plebiszitärer Instrumente}

Außerdem entfalten plebiszitäre Instrumente ihre wichtigsten Vorzüge nicht im einmaligen Gebrauch, sondern durch kontinuierliche Anwendung bzw. Anwendungsmöglichkeit. Also tut man nicht gut daran, ihre praktischen Wirkungen vom Einsatz in seltenen Ausnahmefällen her abzuschätzen. Eher muss man in Staaten mit langer plebiszitärer Tradition wie die Schweiz blicken. Dort entsteht eine „Kultur des Plebiszitären“, die mit Volksabstimmungen nicht brachial wie mit gerade geschenktem Spielzeug, sondern besonnen wie mit einem vertrauten Instrument umzugehen nahelegt. Es ist durchaus nicht verwegen, auch in stabilen repräsentativen Demokratien ohne sonderliche Erfahrungen mit plebiszitären Instrumenten demokratie- und bürgerschaftskultivierende Wirkungen dieser Art zu erwarten, und es ist nicht erforderlich, von vornherein vom schlimmsten Fall auszugehen, wonach plebiszitäre Instrumente ganz einfach Populismus und Demagogie entfesseln oder zu inkonsistenter Politik führen müssen. Gegen beides ist ja auch der Parteienwettbewerb vor und nach Parlamentswahlen nicht gefeit! Welche „Kultur des Plebiszitären“ aber wirklich entsteht, wird im Einzelfall einesteils davon abhängen, ob die eingeführten plebiszitären Instrumente eine für repräsentative Demokratie hilfreiche Ausgestaltung erfahren, und andernteils davon, wie konstruktiv und sinngemäß die für die Prüfung der Zulässigkeit einer je konkreten Verwendung plebiszitärer Instrumente zuständigen Personen und Institutionen bei ihren Entscheidungen und deren Begründung verfahren. 
D. Welche plebiszitären Instrumente sollten wir haben, welche anderen aber nicht?

Dies alles zu wichtigen Rahmenregelungen des Einsatzes plebiszitärer Instrumente vorausgeschickt, lässt sich nun leicht vor Augen führen, welche von ihnen wir gut brauchen könnten, um Responsivität und Führungsarbeit in unserer repräsentativen Demokratie zu verbessern, welche anderen aber wohl zu deren Schaden wirkten.

\section{Sinnvolle plebiszitäre Instrumente}

Durch Volksanträge, mit denen dem Parlament binnen vorgegebener Frist die Vorlage eines Gesetzentwurfs oder von Eckpunkten eines Handlungsprogramms zur Lösung eines Problems auferlegt wird, kann das Volk die politische Klasse dazu zwingen, sich auch mit solchen Problemen zu befassen, die sie - quer über die großen Lager - bislang nicht aufgreifen will. Dieses Instrument lässt sich auch leicht auf der kommunalen Ebene anwenden. Für dieses mildeste aller plebiszitären Instrumente sollte man das geringste sinnvolle Antragsquorum ansetzen. Das könnten sogar weniger als jene fünf Prozent der Wahlberechtigten sein, die ja ihrerseits auch ausreichten, um eine Protestpartei mit ihrem zentralen Anliegen ins Parlament zu wählen. Bislang aber muss man tatsächlich darauf warten, dass eine Protestpartei ein von der politischen Klasse dilatorisch behandeltes Problem - etwa der Zuwanderungs- und Integrationspolitik - aufgreift, und bis anschließend - um die offenbar ein wichtiges Anliegen der Bürgerschaft vertretende Protestpartei wieder kleinzubekommen - die etablierten Parteien sich zu eben jenen Politikkorrekturen bequemen, die sie vorher verweigert haben. ${ }^{30} \mathrm{Ge}-$

30 Das spektakulärste bundesdeutsche Beispiel für das hier zu lösende Problem ist das $\mathrm{Zu}$ sammenspiel zwischen dem Aufstieg der Republikaner und der durch politische Entscheidung herbeigeführten Verringerung des Asylbewerberzuzugs nach Deutschland. Schon in den 1980er Jahre waren viele Deutsche mit der damals noch äußerst liberalen Regelung der Asylgewährung unzufrieden und brachten das, zumal auf kommunaler Ebene, immer wieder zum Ausdruck. Auf der Bundesebene aber scheuten Union und SPD gesetzgeberische Initiativen zur Eingrenzung des Zuzugs von Asylbewerbern. Nach dem Ende des Ost/West-Konflikts stiegen dann die Asylbewerberzahlen rasch auf gut 400.000 im Jahr 1992. Die 1983 neugegründete Partei der Republikaner hatte zuvor schon den Asylbewerberzuzug zu einem ihrer prominenten Themen gemacht und war gerade auch mit ihm bei den Europa- und Landtagswahlen zwischen 1989 und 1992 sehr erfolgreich. So von rechts unter Druck gesetzt, ging die CDU-geführte Bundesregierung endlich das Problem an und wurde von der SPD, die Teile ihrer Stammwählerschaft sich 
rade dieses plebiszitäre Instrument scheinen wir gut brauchen zu können. Einem Prozess der Volksgesetzgebung hat es voraus, dass die Initiatoren sich nicht selbst die Mühe eines Gesetzgebungsvorschlags machen müssen, sondern diese Aufgabe - ganz gemäß den Leitgedanken repräsentativer Demokratie - bei den gewählten Abgeordneten und der von ihnen getragenen Regierung verbleibt. Die politische Klasse kann sich aber der Erfüllung dieser Aufgabe nicht mehr so leicht entziehen wie bislang, zumal dann nicht, wenn obendrein die nachstehend beschriebenen plebiszitären Instrumente eingeführt werden.

Unter ihnen ist in Deutschland die Volksgesetzgebung am bekanntesten. Sie gibt es inzwischen in allen Bundesländern, noch nicht aber auf Bundesebene. Im Bereich des Satzungsrechts könnte sie auch auf kommunaler Ebene eingeführt werden. Sie entfaltet sich am besten in folgenden drei Schritten: Durch Volksinitiative wird die Verabschiedung eines von den Initiatoren vorgelegten Gesetzentwurfs durch das Parlament begehrt; dem kann das Parlament willfahren; und tut es das nicht, so kommt es zu einem Volksentscheid, bei welchem dem durch Volksinitiative vorgeschlagenen Gesetz auch ein Gesetzentwurf der Parlamentsmehrheit zur Seite gestellt werden kann, nicht aber muss. Faktisch handelt es sich hier um eine Steigerung der Möglichkeiten des Volksantrags und um eine klare, vom Volk ausgehende Richtungsvorgabe für die politische Klasse. Sobald häufiger verwendet, dürfte dieses Instrument erhebliche Vorauswirkungen entfalten und die politische Klasse responsiver machen, als sie es ohne das Volk als ,alternativen Gesetzgeber“ wäre. ${ }^{31}$ Das Antragsquorum könnte bei etwa fünf Prozent der Wahl- bzw. Abstimmungsberechtigten angesetzt werden und somit gar niedriger als derzeit in den meisten deutschen Bundesländern, wo obendrein

von ihr auch wegen des ungelösten Asylbewerberproblems entfremden sah, bei der dafür nötigen Grundgesetzänderung von 1993 unterstützt. Kurze Zeit später schmolz dann wieder das Wählerpotential der Republikaner. Vermutlich hätte, mit dem Risiko eines Volksantrags oder gar mit diesem selbst konfrontiert, die politische Klasse bereits in den 1980er Jahren das Asylbewerberproblem angegangen. Dann wäre unserem Land das Selbstverständlichwerden rechtsradikaler Protestparteien erspart geblieben, aktive $\mathrm{Zu}$ wanderungs- und Integrationspolitik schon eher auf die Agenda gekommen und hätte unsere Gesellschaft heute weniger Integrationsprobleme.

31 In der deutschen Verfassungspraxis leidet dieses Instrument freilich an zwei Problemen, die es weitgehend um seinen Nutzen bringen. Erstens wurden in einigen Bundesländern so hohe Antrags- und Beteiligungsquoren eingeführt, dass die Chancen auf eine erfolgreiche Nutzung dieses plebiszitären Instruments abschreckend gering sind. Zweitens entzieht der Ausschluss von finanzwirksamen Vorlagen wichtige Themen der Bearbeitung mit dem Instrument der Volksgesetzgebung. Derzeit noch sehr unzulänglich ausgestaltet, wirkt das Instrument der Volksgesetzgebung heute so, als sei es zur Verbesserung repräsentativer Demokratie wenig brauchbar. Nichts hindert aber daran, es zu verbessern. 
die - hier ausdrücklich nicht empfohlenen - Beteiligungs- oder Zustimmungsquoren $^{32}$ die Wirksamkeit von Volksgesetzgebung stark verringern. Für deren Abschaffung spricht im Übrigen auch, dass die Volksgesetzgebung an sich schon ein gegenüber dem parlamentarischen Gesetzgebungsverfahren erschwertes Gesetzgebungsverfahren ist. Hier gilt es nämlich fallweise erst einmal die Hürde des Antragsquorums zu überwinden, während ein Parlament jederzeit die Gesetzesinitiative ergreifen kann.

Etwa in der Schweiz, nicht aber - abgesehen von Rheinland-Pfalz und Hamburg - in Deutschland, ist das plebiszitäre Instrument des fakultativen Gesetzesreferendums bekannt. Man kann zu ihm greifen, wenn das Parlament ein Gesetz beschlossen hat, ein nennenswerter Teil der Bürgerschaft dieses Gesetz aber zu korrigieren oder abzuschaffen wünscht. Bislang gibt es nur zwei Lösungen dieses Problems. Erstens kann die parlamentarische Opposition vor dem Verfassungsgericht ein abstraktes Normenkontrollverfahren anstrengen, womit politische Gestaltungswünsche aber Richtern angetragen werden. Zweitens kann die parlamentarische Opposition ankündigen, das wider ihren Willen beschlossene Gesetz zum Gegenstand des nächsten Wahlkampfs zu machen und es nach eigenem Wahlsieg wieder abzuschaffen. Das freilich verschiebt die Problemlösung bis über das Ende der Wahlperiode hinaus. Viel einfacher ließe sich eine zeitnahe Problemlösung mit einem fakultativen Gesetzesreferendum erzielen.

Dessen Leitgedanke besteht darin, im Fall eines inhaltlichen Konflikts zwischen Parlamentsmehrheit und artikulationsstarken Teilen der Bevölkerung eine ausdrückliche Bestätigung des umstrittenen Gesetzes durch eine Volksabstimmung zu verlangen. Erhält das Gesetz dabei keine Mehrheit, tritt es binnen bestimmter Frist außer Kraft. Die unmittelbare Auswirkung einer Verfügbarkeit dieses Instruments auf die politische Klasse geht dahin, dass sie Gesetze nun nicht nur „verfassungsgerichtsfest“ machen muss, also so auszugestalten hat, dass ein Gesetz ein abstraktes Normenkontrollverfahren möglichst unverändert übersteht. Sondern nun muss ein Gesetz auch noch „referendumssicher“ sein, d.h. ein möglichst geringes Risiko in sich tragen, dass ein nennenswerter Teil der Bevölkerung seine Aufhebung verlangt, sodann die Hürde des Antragsquorums übersprungen wird und am Ende noch eine „Volksabstimmung gegen das Gesetz“ gelingt. Einesteils spricht die Plausibilität des ersten Anscheins dafür, es solle das Antragsquorum hier dasselbe wie bei der Volksgesetzgebung sein, also bei fünf Prozent der Wahl- bzw. Abstimmungsberechtigten liegen. Andernteils kann man sich ans beliebte Bild von „Gaspedal“ und „Bremse“ halten: Manche 
plebiszitären Instrumente - zumal Volksanträge und Volksgesetzgebung, doch auch eine plebiszitär herbeigeführte Parlamentsauflösung - wirkten wie ein vom Volk betätigtes Gaspedal, während andere, allen voran die fakultativen Referenden - dem Volk aktives Bremsen erlaubten. In dieser Sicht ist es angebracht, immer dann schon vorab mehr Unterstützung zu verlangen, wenn eine antragstellende Minderheit den bestehenden Zustand verändern will, und dann weniger Unterstützung, wenn eine antragstellende Minderheit den status quo aufrechterhalten will, nämlich durch Aufhebung jener Parlamentsentscheidung, die ihrerseits den bestehenden Zustand verändert. Ob man einen - außerdem: wie großen - Unterschied zwischen den Antragsquoren bei Volksanträgen bzw. Volksgesetzgebung einerseits sowie fakultativen Gesetzesreferenden andererseits machen will, hängt also ganz davon ab, wie umfangreich man das ,konservative Potential“" plebiszitärer Instrumente auszugestalten wünscht. Die gleiche Überlegung prägt - neben dem Kurzhalten von Zeiten der Rechtsunsicherheit - auch die Antwort auf die Frage, wie lange jene Frist währen soll, in welcher es nach Verabschiedung eines Gesetzes das Antragsquorum zu erreichen gilt, um ein fakultatives Gesetzesreferendum auf den Weg zu bringen. Einmal anwendungsfähig institutionalisiert, hält gerade das fakultative Referendum die Repräsentanten zu erheblicher Responsivität sowie - bei den Kampagnen um die Erreichung des Antrags- und später vielleicht eines Zustimmungsquorums - zu viel größeren kommunikativen Führungsleistungen an, als sie andernfalls zu erwarten wären. Gerade das dient repräsentativer Demokratie.

Zügige Reformpolitik wird durch fakultative Gesetzesreferenden zwar nicht gefördert. Doch derlei ist - wie etwa der Aufgeklärte Absolutismus zeigt - ohnehin eher die Domäne wohlmeinender Autokraten. Also ist es unangebracht, schnelles Befinden über weitreichende Reformen ausgerechnet von Demokratien zu verlangen. Außerdem werden sich gerade schmerzliche Reformen dann leichter durchsetzen lassen, wenn sich nachweislich keine Mehrheit gegen sie findet. Diesem Nachweis dient aber nichts besser als ein fakultatives (Verhinderungs-) Referendum, das zunächst von einer öffentlichkeitsträchtigen Minderheit auf den Weg gebracht wird und anschließend bei der - sonst zum Schweigen verurteilten, beim Referendum aber mit vernehmbarer Stimme ausgestatteten - Mehrheit keine Zustimmung findet.

Sollten die Repräsentierten nicht auch noch - und zumal oberhalb der kommunalen Ebene - das Recht erhalten, über ihre fallweise Mitwirkung an der Gesetzgebung hinaus Einzelfragen zu entscheiden? Das kann man bejahen, sofern sichergestellt ist, dass dieses plebiszitäre Instrument strikt „,von unten nach oben“ wirkt und keinesfalls die politische Klasse ein Mittel bekommt, eigene Entscheidungsverantwortung ,von oben nach unten“, also auf die Abstimmenden, abzu- 
wälzen. Die Regel lautet somit wie beim fakultativen Gesetzesreferendum: Das erste Wort müssen die Repräsentanten haben, das letzte Wort aber die Repräsentierten. Und besitzt das Volk auch noch das Instrument des Volksantrags, so kann es obendrein seinen Vertretern jenes erste Wort abverlangen.

Dieser Regel folgend, sollte das Verfahren einer ,Vorlageninitiative“ so ablaufen: Erst einmal hat das Parlament bzw. die parlamentsgetragene Regierung gegebenenfalls durch Volksantrag dazu aufgefordert - einen Vorschlag zur Lösung eines Problems zu unterbreiten, beispielsweise über die Eckpunkte einer Schulreform oder über den Beitritt eines Landes zur EU; sodann kann das Volk begehren, dass ihm diese Entscheidung zur Billigung vorgelegt wird; und wird das - wohl wiederum bei fünf Prozent der Wahl- bzw. Abstimmungsberechtigten anzusetzende - Antragsquorum erreicht, so findet eine Volksabstimmung statt. Deren zunächst einmal verbindliches Ergebnis kann nötigenfalls in den oben beschriebenen Weisen wieder korrigiert werden: entweder durch eine erneute, auf Abänderung ausgehende Vorlageninitiative, oder durch den verändernden Beschluss eines - gegebenenfalls nach Selbstauflösung - neu gewählten Parlaments.

In Verbindung mit einem vorgängigen Volksantrag vermag die Bürgerschaft auf diese Weise jede weichenstellende Entscheidung an sich zu ziehen, und zwar ganz ohne die Repräsentanten je aus ihrer Erstverantwortung zu entlassen. Natürlich kann dieses Instrument auch leicht von politischen Parteien mit ihrer großen Mobilisierungsfähigkeit genutzt werden und erlaubt dann der Opposition Korrekturversuche der Regierungspolitik, und zwar ganz ohne das in den letzten Jahren so häufig genutzte Instrument des abstrakten Normenkontrollverfahrens. Das wiederum reduzierte die dem Bundesverfassungsgericht im Lauf der Zeit zugewachsene genuin politische Macht, die ja notwendigerweise aus abstrakten Normenkontrollverfahren zu zentralen parteipolitischen Streitfragen fließt, auf das rechtsstaatlich wirklich Gebotene.

Obendrein könnten obligatorische Referenden $\mathrm{zu}$ vorab verfassungsmäßig festgelegten Entscheidungsmaterien die politische Klasse zu mehr Responsivität und insgesamt zu besseren Führungsleistungen anhalten. Zum einen dürften Verfassungsreferenden sinnvoll sein. Sie senkten nämlich das Risiko, dass Verfassungstexte immer detaillierter und zeitbezogener werden. Gerade dazu führt nämlich das Streben der politischen Klasse, den politischen Gegner bei schwierigen Entscheidungen unterstützungssichernd „einzubinden“. Zu diesem Zweck sichert man einzelnen Oppositionsfraktionen zu, gewünschte Maßnahmen nicht ohne Verfassungsänderung zu ergreifen. Wegen der dadurch drastisch erhöhten Mehrheitsanforderung wird der bei einfacher Abstimmungsmehrheit leicht zu besiegende politische Gegner nun zum legitimen „Veto-Spieler“. Ihm kommt 
man dann - gerade gegen Widerstand in den eigenen Reihen - soweit entgegen, dass ein gemeinsam getragener Kompromiss erreicht wird; und für diesen erwartet man dann auch von der solchermaßen einbezogenen Opposition verlässliche Unterstützung angesichts öffentlicher Kritik. Das ist ein vorzüglicher Weg, um zu legitimitätssichernden Entscheidungen zu gelangen. Doch der Bürgerschaft bleibt angesichts solcher Tauschgeschäfte innerhalb der politischen Klasse nur die Zuschauerrolle. Wenn hingegen jede Verfassungsänderung anschließend einem obligatorischen Referendum unterzogen werden muss, dann setzt das solchen Insidergeschäften recht enge Grenzen öffentlicher Plausibilität.

Zum anderen kann man überhaupt einen Katalog von Themen formulieren und verfassungsmäßig verankern, die dem Volk so wichtig sind, dass es vor dem Zustandekommen einer entsprechenden Regierungsmaßnahme oder dem Beschluss eines einschlägigen Gesetzes unbedingt nach seinem Urteil gefragt werden möchte und in diesem Fall der Parlamentspräsident - nicht aber der Regierungs- oder Staatschef! - ohne eigenen Ermessensspielraum ein Referendum ansetzen muss. Im deutschen Fall könnten die Übertragung nationaler Souveränitätsrechte auf die EU oder die Aufnahme weiterer Staaten in die EU zu Gegenständen solcher obligatorischer Referenden gemacht werden, weil sie zweifellos die Lage und Zukunft des eigenen Landes und Volks nachhaltig betreffen. Wenn mit der einmaligen ermessensfreien Ansetzung eines solchen Referendums die Möglichkeit dann auch ausgeschöpft ist, eine entsprechende Frage ,von oben nach unten“ dem Volk vorzulegen, ist zugleich ausgeschlossen, dass man das Volk einfach solange abstimmen lässt, bis sich das politisch gewünschte Ergebnis einstellt. Stattdessen sollte obendrein vorgesehen werden, dass nur über eine Vorlageninitiative das Ergebnis einer obligatorischen Volksabstimmung wieder abgeändert werden kann.

Weil es bei obligatorischen Referenden keine Antragsquoren gibt, wird mancher hier „zum Ausgleich“ an ein besonders hohes Beteiligungsquorum denken. Doch der oben umrissene Wirkungszusammenhang besteht ja auch bei obligatorischen Referenden: Sobald es ein Beteiligungsquorum gibt, kann man ,gegen die Abstimmungsbeteiligung Wahlkampf machen“, ist also nicht gehalten, sich wirklich auf den Entscheidungsgegenstand einzulassen. Solches Ausweichen vor der tatsächlichen Entscheidungsfrage gilt es aber der Demokratie willen zu unterbinden. Im Übrigen wirkten hohe Beteiligungsquoren bei obligatorischen Referenden ganz einfach zugunsten des status quo, was mit Blick auf immer wieder nötigen Reformbedarf wenig wünschenswert erscheint. Will man aus solchen Gründen auf ein Beteiligungsquorum verzichten, mag man dennoch ein Zustimmungsquorum für sinnvoll halten. Es leuchtet ja die Durchführung eines obligatorischen Referendums mit Bindewirkung dann nicht ein, wenn nur ein ver- 
schwindender Prozentsatz der wahlberechtigten Bevölkerung der auf breite Akzeptanz zu stellenden Regelung zustimmt. Weil obligatorische Referenden nur bei wichtigen Themen in Anwendung kommen, könnte man deshalb hier an ein Zustimmungsquorum von fünf Prozent der Wahlberechtigten denken. Gerade bei obligatorischen Verfassungsreferenden liegt ein Zustimmungsquorum systematisch nahe, weil bei Verfassungsänderungen ja auch im Parlament ein durch höhere Hürden als die einfache Mehrheit erschwertes Gesetzgebungsverfahren greift.

Das durchschlagendste Instrument, um auch eine solche Parlamentsmehrheit zur Responsivität zu zwingen, die sich in einem der Bevölkerung wichtigen Punkt absichtlich irresponsiv verhält oder systematisch vom artikulierten Mehrheitswillen der Repräsentierten abweicht, ist die plebiszitär herbeigeführte Auflösung des Parlaments. Mit diesem Instrument - idealerweise unnötig und in der Praxis durch Nutzung der anderen plebiszitären Instrumente entbehrlich zu machen - könnte das Volk sowohl auf die es nicht befriedigende Behandlung eines Volksantrags reagieren als auch eine solche Parlamentsmehrheit in Neuwahlen schicken, die in der Bevölkerung dauerhaft keine Unterstützung mehr findet. Natürlich darf dieses Instrument nie vorschnell oder spielerhaft einsetzbar sein, weil doch die ganze Konfliktfähigkeit der Repräsentanten von der Sicherheit ihres Mandats abhängt. Also muss man beim Antragsquorum für eine plebiszitär herbeizuführende Parlamentsauflösung an die Obergrenze des Sinnvollen gehen. Die Maxime muss wohl sein, dass dieses Instrument zwar grundsätzlich verwendbar sein soll, doch tatsächlich nur nach einer wirklich großen politischen Anstrengung eingesetzt werden kann, deren Erbringung von einem wirklich bestehenden - und nicht nur im pluralistischen Streit behaupteten - Repräsentationsdefizit zeugt.

Also wird man das Antragsquorum plausiblerweise an jenem Rückhalt bemessen, den die amtierende Regierungsmehrheit bei ihrem Amtsantritt im Volk hatte. Die Leitidee geht dahin, dass man eine Regierungsmehrheit wohl dann ins Risiko von Neuwahlen schicken darf, wenn der Anteil derer, die Neuwahlen herbeiführen wollen, mindestens so groß ist wie der Anteil jener, die bei der Regierungsbildung hinter der Regierung standen. Deshalb errechnet sich das im konkreten Fall zu überwindende Antragsquorum aus der Wahlbeteiligung der letzten Wahl sowie dem Wähleranteil der regierungstragenden Parteien. Liegt dieser beispielsweise bei 60 Prozent, so stehen bei einer Wahlbeteiligung von 50 Prozent nicht mehr als 30 Prozent der Bevölkerung hinter der regierungstragenden Parlamentsmehrheit. Wenn unter diesen Umständen ebenfalls 30 Prozent der Bevölkerung die Auflösung des Parlaments begehren, lässt sich demokratietheoretisch wenig dagegen einwenden, dass es anschließend zur Volksabstimmung 
über die Parlamentsauflösung kommt. Ein Vorzug dieser Festlegung des Antragsquorums liegt neben seiner inhaltichen und gerade nicht bloß formalen Begründung darin, dass die Repräsentanten selbst durch eine Regierungsneubildung unter Erweiterung der Regierungsmehrheit die Überlebenschancen ihres Parlaments steigern können, während sie bei Koalitionszerfall aufgrund mangelnder Führungskraft, und zumal beim Zustandekommen nur einer Minderheitsregierung, ihrerseits das eigene Risiko steigern, vor den Wähler als Kadi gezogen $\mathrm{zu}$ werden. Eben solche Zusammenhänge praktisch nutzbar zu machen, entspricht ganz der Funktionslogik repräsentativer Demokratie. ${ }^{33}$ Wie im Fall eines obligatorischen Referendums ließe sich bei diesem Instrument an ein Zustimmungsquorum denken. Man könnte es bei 50 plus 1 Prozent des jeweiligen Antragsquorums festsetzen, um dieses Instrument nach Überwindung des ohnehin hohen Antragsquorums scharf zu halten, zugleich aber nicht einer kleinen Minderheit die Auflösung des Parlaments zu erlauben.

\section{Abzulehnende plebiszitäre Instrumente}

Gemeinsam ist allen vorgeschlagenen plebiszitären Instrumenten (Volksantrag, Volksgesetzgebung, fakultatives Gesetzesreferendum, Vorlageninitiative, obligatorisches Referendum, plebiszitäre Parlamentsauflösung), dass sie „,vom Volk hin zu den Repräsentanten“ und sozusagen ,von unten nach oben“ wirken. Das muss in einer Demokratie auch genau so sein. Jedes der genannten Instrumente ist zudem geeignet, das Funktionieren repräsentativer Demokratie in der oben beschriebenen Weise zu verbessern. Also könnten wir sie wirklich brauchen. Hingegen brauchen wir keine plebiszitären Instrumente, die aufgrund ihrer Funktionsweise und Nebenwirkungen die repräsentative Demokratie zu schädigen drohen. Abzulehnen sind vor allem jene plebiszitären Instrumente, die politische Verantwortung von der politischen Klasse wegverlagern können oder gar jene politischen Kommunikationsprozesse, die eine Abstimmungskampagne nun einmal begleiten, zielgenau zum persönlichen Machtgewinn für die politische Elite nutzbar machen.

33 Man könnte systematisch sogar noch weiter ausholen: Im parlamentarischen Regierungssystem gehören die Bestellung des Regierungschefs durch das Parlament und das Recht des Regierungschefs auf Parlamentsauflösung eng zusammen, wird doch so wechselseitig Macht ausbalanciert. In ähnlicher Weise scheinen in einem demokratischen Repräsentativsystem nun aber auch das Führungsrecht der Repräsentanten und Parlamentsauflösungsrecht der Repräsentierten inhaltlich zusammenzugehören. 
Besonders schlecht für repräsentative Demokratie, ja sogar für die Demokratie selbst, ist ausgerechnet das bekannteste plebiszitäre Instrument: das von Verfassungsorganen initiierte fakultative Referendum. Dieses wird so gehandhabt, dass eine Parlamentsmehrheit, eine Regierung durch Mehrheitsbeschluss oder ein Regierungs- bzw. Staatschef aus eigenem Ermessen eine Volksabstimmung zu einer aktuellen oder für aktuell erklärten Frage ansetzt. Die Leitidee dieses Instruments kann zwar demokratischer gar nicht sein: Das Volk wird gefragt, wie ein Sachproblem gelöst werden oder wie eine Entscheidung ausfallen soll. Doch die Nebenwirkungen der praktischen Umsetzung dieser Leitidee sind höchst riskant.

Erstens hat dieses plebiszitäre Instrument zwar alles mit Demokratie, nichts aber mit Repräsentation zu tun und verschenkt deren Mehrwert. In einem Repräsentativsystem ist es nämlich - wie oben dargelegt - zunächst einmal nicht nur das Recht, sondern vor allem die Pflicht der Parlamentarier bzw. der parlamentsgetragenen Regierung, und im präsidentiellen Regierungssystem auch die des Staatsoberhaupts, Lösungsmöglichkeiten für anstehende Probleme auszuarbeiten und im Rahmen des doch erteilten freien Mandats eigenständige Entscheidungen zu treffen. Natürlich hat das Volk grundsätzlich das Recht, durch die Möglichkeiten politischer Partizipation - möglichst einschließlich plebiszitärer Instrumente - eigene Impulse ins politische System hineinzutragen und seine Repräsentanten bei der nächsten Wahl mit dem Stimmzettel zu bestrafen. Doch vor allem hat das Volk in einem Repräsentativsystem das Recht darauf, sich nicht selbst zu komplexen Themen eine Position erarbeiten zu müssen, sondern dergleichen von seiner - im Übrigen auch gar nicht schlecht bezahlten - politischen Klasse angeboten zu bekommen. Also ist es pflichtwidrig, wenn die Repräsentanten aus Scheu vor einer politischen Niederlage der Bürgerschaft schon gar nicht mit einer eigenen Erstentscheidung zu einem schwierigen Problem kommen.

Und tatsächlich neigt die politische Klasse immer wieder dazu, Entscheidungen mit ungewissen oder unerwünschten Folgen entweder nicht zu treffen oder sofern nur irgend möglich - in Form regierungsinitiierter Sachreferenden an das Volk abzuschieben. Sogar im allen plebiszitären Instrumenten so abgeneigten Bundestag erwog man 1991 allen Ernstes eine Volksabstimmung zur Frage, ob das Parlament wohl in Bonn bleiben oder seinen Sitz in Berlin nehmen solle. Vor diesem Erfahrungshintergrund stelle man sich vor, Entscheidungen über die Weiterentwicklung unseres Gesundheitswesens, über Reformen unseres Sozialversicherungssystems oder über Weichenstellungen unseres Energieversorgungssystems ließen sich auf diese Weise ans Volk abschieben und - einer risikoscheuen politischen Klasse höchst willkommen - aus den Wahlkämpfen heraushalten: Rasch erhielte man dann eine „Politik des geringsten Widerstandes“, die 
sich zudem als „besonders demokratisch“ ausgäbe. Obendrein wäre mit besonders vielen sachlichen Fehlentscheidungen zu rechnen, da vor allem die taktischen Erwägungen von Spitzenpolitikern Inhalt, Tragweite und Zeitpunkt der Volksabstimmungen prägen würden. Die Folgen einer alsbald eher schlechten Regierungsführung müsste sich - wie bei Wahlen - das Volk selbst zurechnen; die politische Klasse hingegen könnte ihre Hände stets in Unschuld waschen: Sie habe ja nur den Bürgerwillen festgestellt und dann vollzogen, könne also wirklich nichts dafür, wenn dieser - immerhin gut demokratisch - auf leider Nachteiliges ausgegangen sei. Die zentralen Vorteile eines Repräsentativsystems - nämlich Leistungsdruck auf die politische Klasse bei responsivitätssicherndem persönlichem Karriererisiko für Spitzenpolitiker - wären auf diese Weise verspielt.

Zweitens wären, wie der Blick in Geschichte und Gegenwart lehrt, im Fall von staatlich initiierten Sachreferenden Tür und Tor für manipulative Formulierungen der Abstimmungsfragen und für hieraus entstehende legitimitätsgefährdende Sekundärkonflikte geöffnet. Obendrein könnte die politische Klasse jene komplizierten Prozesse des Interessenausgleichs leicht umgehen, die den ja auch im Parlament üblichen Ja/Nein-Abstimmungen zugrunde liegen. Im Parlament kann man nämlich Zugeständnisse bei Kompromissen quer über mehrere $\mathrm{Ab}$ stimmungen ,verrechnen“, weil dort dieselben Personen jahrelang zusammenarbeiten; bei Volksabstimmungen aber steht wegen der Anonymität der Abstimmenden jedes Plebiszit für sich.

Drittens - und vor allem - schützt kaum etwas dagegen, dass mit ,von oben nach unten wirkenden“ plebiszitären Instrumenten ganz taktisch zum Zweck persönlicher Machtsicherung verfahren wird. „Plebiszitärer Cäsarismus“ ist der ganz angemessene Name für diesen institutionellen Mechanismus. Wenige Beispiele genügen, ihn zu illustrieren. Mehrfach schon ließ ein Präsident mit zeitlich befristetem Amt sich durch ein selbst initiiertes Referendum zum Staatschef auf Lebenszeit wählen und schlug in unverfroren scheindemokratischer Weise damit den Weg zur Diktatur ein. Oder es wird im Gewand eines Sachreferendums und gerade nicht mittels einer Wahl - dem Volk die Abstimmung über einen Spitzenpolitiker abverlangt. So hielt es General de Gaulle 1962, als das von ihm initiierte Referendum zur Einführung der Direktwahl des Staatspräsidenten für jedermann verständlich dieselbe Frage formulierte wie 1969 das wiederum von ihm angesetzte Referendum über die Reform der Regionalverwaltung und des Senats: „Wollt Ihr mich an der Spitze des Staates?“ 1962 machte das Referendum de Gaulle zum republikanischen Monarchen - und 1969 schickte es ihn in den politischen Ruhestand. Was auf den ersten Blick wie Demut der Regierenden vor dem Volk anmutet, erweist sich auf den zweiten Blick somit als zusätzliches Instrument persönlicher Machtsicherung. Gefährlich auch noch für die De- 
mokratie wird es dann, wenn sich ein Spitzenpolitiker auf diese Weise zielgenau ein Mandat zum Überspielen oder Beiseitedrängen zunächst des Parlaments und anschließend von Rechts- und Verfassungsnormen verschafft. „Bonapartismus“ ist der eingeführte Name für eine solche auf - oft manipulierten - Volkswillen rekurrierende Diktatur.

Das alles vor Augen, ist nicht zu erkennen, wodurch von der politischen Klasse initiierte Referenden einer Demokratie dienlich sein sollten. Anders als durch gedankenlose Lust am eigenen Entscheidenkönnen oder durch mangelnden Einblick in die Funktionslogik politischer Prozesse ist kaum zu erklären, warum Forderungen nach ausgerechnet solchen Referenden so populär sind. Denn tatsächlich braucht es von der politischen Klasse angesetzte Sachreferenden gar nicht: Sofern das oben beschriebene plebiszitäre Instrumentarium verfügbar ist, besitzt das Volk ohnehin wirkungsvolle Möglichkeiten, Entscheidungen der Repräsentanten zu korrigieren und seine Vertreter, um die Auswirkungen plebiszitärer Instrumente wissend, von vornherein zur Responsivität anzuhalten. Aktive Teile der Bürgerschaft haben dann obendrein alle Möglichkeiten, über Volksantrag und Vorlageninitiative, auch über die Volksgesetzgebung und letztlich über die Drohung mit einer plebiszitär bewirkten Parlamentsauflösung überall dort selbst politisch gestaltend tätig zu werden, wo ein ,,von oben angesetztes“ fakultatives Sachreferendum das Volk doch bloß vor eine Alternative stellte, welche die politische Klasse ausgearbeitet hat: für oder gegen die X-Reform, für oder gegen die Y-Politik, für oder gegen die Maßnahme Z. Ein Volk, das anstelle der hier vorgeschlagenen plebiszitären Instrumente auf Politikerinitiative zurückgehende fakultative Sachreferenden erhielte, machte also ein wirklich schlechtes Geschäft.

Ganz überflüssig und außerdem politisch sehr nachteilig sind „,konsultative Referenden ", also solche sachunmittelbaren Abstimmungen, die von der politischen Klasse unter der Maßgabe angesetzt werden, sie würden ohnehin keine Bindewirkung entfalten. ${ }^{34} \mathrm{Um}$ die Präferenzen der Bevölkerung ausfindig zu machen, braucht es sie wirklich nicht; dies leisten demoskopische Umfragen viel genauer und billiger. Doch Regierende können konsultative Referenden benutzen, um der Opposition in aller Öffentlichkeit ihre Machtlosigkeit zu demonstrieren und anschließend auf die Sogkraft der Mehrheitsposition und Mehrheitspartei

34 An die Grenze politischer Perversion führte es, wenn man ein konsultatives Referendum ansetzte, zugleich aber ankündigte, man werde dessen Ergebnis als verbindlich behandeln. Derlei setzt nämlich einen Prozess der Informalisierung des Politischen in Gang, an dessen Ende die Akzeptanz ganz willkürlichen Umgangs mit politischen Spielregeln stehen wird. 
zu hoffen. Sie können auch mit einer willkürlich entfachten Referendumskampagne von anderen politischen Themen oder ihnen lästigen Problemen ablenken. Und in der Hand von Regierenden können konsultative Referenden ebenfalls der Entlastung von unangenehmen Gestaltungsaufgaben dienen: Stimmt das Volk nicht schon vorab bestimmten Reformhärten zu, dann muss man sich auch nicht in das Risiko eines Konflikts mit dem Volk begeben - und wird obendrein nicht für Tatenlosigkeit gescholten, sondern ob vorbildlicher demokratischer Haltung gelobt.

\section{Irreführende Klassifikationen „direkter Demokratie“}

Offenbar ist also ein konsultatives Referendum etwas anderes als ein fakultatives Gesetzesreferendum und eine Vorlageninitiative etwas anders als ein von Verfassungsorganen initiiertes Referendum. Dann geht freilich auch die populäre Gegenüberstellung von „Referendumsdemokratie“ und „Volksgesetzgebung“ an den wirklich zu bedenkenden Zusammenhängen völlig vorbei. Die zentrale Scheidelinie verläuft vielmehr zwischen plebiszitären Instrumenten, die allein in der Hand der politischen Klasse liegen, also „,von oben nach unten“ wirken, und solchen, deren Verwendung von Bürgergruppen - und darunter natürlich auch den Parteien - initiiert werden kann, also politische Wirkungen ,,von unten nach oben“ entfaltet. Die erstgenannten plebiszitären Instrumente tun jeglicher Demokratie unausweichlich schlecht, die letzteren der repräsentativen Demokratie vermutlich sogar gut. Eben diese Einsicht sollte jede Parteinahme für die eine oder andere Instrumentengruppe prägen.

Um eine ganz andere Thematik geht es hingegen bei der Frage, ob Ministerpräsidenten und Bundeskanzler - so wie inzwischen allenthalben die Bürgermeister und Landräte - direkt vom Volk gewählt werden sollten. ${ }^{35}$ Hier handelt es sich nämlich nicht um eine Anreicherung repräsentativer Demokratie um plebiszitäre Instrumente, sondern um die Ausgestaltung des Regierungssystems: Soll es ein präsidentielles oder ein parlamentarisches sein? Im ersten Fall sind Direktwahlen der Staats- bzw. Regierungschefs vorzusehen und ist nach Möglichkeit zu vermeiden, dass es zu einer „doppelten Exekutive“ kommt, also zum - oft „semi-präsidentielles Regierungssystem“ genannt - gemeinsamen Bestehen der Ämter von Staats- und Regierungschef. Im zweiten Fall darf man hingegen gera-

35 Siehe hierzu W.J. Patzelt, Unsere Verfassung verträgt keine Basteleien, in: FAZ Nr. 128 v. 5.6.2009, 9 . 
de keine Direktwahl von Staats- oder Regierungschefs befürworten, weil die Realisierung dieses Wunsches ohne vollständigen Übergang zum präsidentiellen Regierungssystem das ganze Staatswesen anschließend oft in schlimme Funktionsprobleme stürzt. Im Übrigen ist es nicht nötig, dass in einem Mehr-EbenenRegierungssystem auf allen Systemebenen derselbe Typ von Regierungssystem besteht. Unser eigenes Land lebt beispielsweise recht gut damit, dass auf der kommunalen Ebene präsidentielle, auf Landes- und Bundesebene hingegen parlamentarische Regierungssysteme arbeiten.

Es ist im Übrigen höchst nachteilig für die deutsche Debatte um plebiszitäre Instrumente, dass unter dem populären Begriff der „direkten Demokratie“ zusammengezogen wird, was offensichtlich strikt voneinander zu trennen ist: die Festlegung des Regierungssystems und die Einführung von plebiszitären Instrumenten mit Wirkung ,von unten nach oben“ bzw. ,von oben nach unten““. ${ }^{36} \mathrm{Au}$ ßerdem lassen sich gute Argumente für die Position finden, dass präsidentielle Regierungssysteme, in denen der Regierungschef nicht vom Parlament aus seinem Amt entfernt werden kann, zur Sicherung ihrer Responsivität in viel größerem Maße plebiszitärer Instrumente bedürfen als parlamentarische Regierungssysteme, die schon von ihrer Funktionslogik her viel sensibler auf Umschichtungen in den Wählerpräferenzen reagieren. Vor diesem Hintergrund muss dann auch nicht wundern, wenn die politischen Systeme mit der umfangreichsten plebiszitären Praxis - nämlich Kalifornien und die Schweiz - entweder ein präsidentielles Regierungssystem haben oder, wegen der Unabsetzbarkeit der Regierung, wie ein präsidentielles Regierungssystem funktionieren. Alle diese wichtigen Differenzierungen werden aber ausgeblendet, wenn man ganz diffus von ,direkter Demokratie“ spricht.

\section{E. Was darf man von (mehr) plebiszitären Instrumenten erwarten - und was nicht?}

Gemäß den vorgetragenen Grundsätzen eingeführte plebiszitäre Instrumente werden die repräsentative Demokratie nicht schädigen, sondern verbessern. Sie garantieren gleichwohl weder gutes Regieren noch die rasche Lösung selbst dringlicher politischer Probleme. Im Gegenteil erhöhen sie mancherlei politische

36 Siehe hierzu W.J. Patzelt, Populäre Missverständnisse „direkter Demokratie“ als Herausforderungen von Politik und politischer Bildung, in: P. Neumann/D. Renger (Hrsg.), Sachunmittelbare Demokratie im interdisziplinären und internationalen Kontext 2008/2009. Deutschland - Österreich - Schweiz, 2010, S. 211-223. 
Transaktionskosten, weil sie die Macht der inner- und außerparlamentarischen Opposition stärken und somit das Regieren schwerer machen. Vor allem dürften sich plebiszitäre Instrumente vielfach entschleunigend auswirken: Reformen akzeptiert man plebiszitär erst dann, wenn es wirklich keine mehrheitsfähigen Argumente gegen sie mehr gibt. Doch solche Entschleunigungswirkungen mögen plebiszitäre Instrumente in einer ohnehin schnelllebigen und zum politischen Aktionismus verleitenden Zeit sogar besonders attraktiv erscheinen lassen, zumal ihre Anwendung ja nicht den Standardmodus politischer Entscheidungsfindung, sondern deren - durch gewisse Häufigkeit aber ganz undramatischen - Ausnahmefall darstellen wird. Ohnehin muss man sich ob einer „Übernutzung“ plebiszitärer Instrumente keine großen Sorgen machen: Sie alle verlangen nach einem großen Einsatz an Zeit, Energie und Geld, was in der Regel erst dann zu ihnen greifen lässt, wenn hinter ihnen ein echtes und darum ernstzunehmendes Anliegen steht. Und weil mit einer plebiszitären Niederlage meist auch Einbußen an Ansehen und Macht verbunden sind, werden gerade erfolgsorientierte Initiatioren ihr Risiko vorab achtsam abschätzen.

Schon gar nicht sind plebiszitäre Instrumente geeignet, die Macht politischer Parteien zu beschneiden. Für einen solchen Wunsch gibt es auch keinen wirklich guten Grund. Ohne Parteien lassen sich nämlich weder freie Wahlen durchführen noch machtvolle Parlamente betreiben. Deshalb wird es ganz in Ordnung sein, wenn sich - wie zu erwarten ist - gerade auch die Parteien an die Nutzung plebiszitärer Instrumente machen. Doch sie werden dabei leichter Konkurrenz bekommen, und zwar nicht nur - wie bislang in der politischen Willensbildung und Entscheidungsfindung - durch ihnen an demokratischer Legitimität weit unterlegene Interessengruppen und Massenmedien, sondern vielmehr durch Akteure ihresgleichen: Vor Zulassungsbehörden und Antragsquoren sind nämlich Parteien und sonstige Antragsteller auf Nutzung eines plebiszitären Instruments völlig gleich.

Gewiss ist nach Einführung wirklich nutzbarer plebiszitärer Instrumente die Entstehung einer Art „Referendumsindustrie“ sowie einer Gruppe von professionellen „Referendumsinitiatoren“ zu erwarten. Das gilt zumal für Mediendemokratien, deren Funktionslogik rasch auch plebiszitäre Prozesse prägen wird. Doch da es längst schon Berufspolitiker gibt, stellt dergleichen nur eine Art Waffengleichheit zwischen der politischen Klasse und den sich nur fallweise ins politische Gewerbe begebenden Bürgergruppen dar. Natürlich werden diese, ins Magnetfeld praktischer Politik und Medienlogik geraten, rasch ihren vielleicht anfangs durchaus noch gegebenen „basisdemokratischen“ und ,amateurhaften“ Charakter verlieren; stattdessen wird eine Art „plebiszitäres Landsknechtwesen“ entstehen, das sich obendrein seine Auftraggeber sucht. In der solches Treiben 
alsbald begleitenden Protestfolklore wird es außerdem manch unschöne Verquickungen persönlicher Eitelkeit, hintergründigen Erwerbsstrebens und bloß funktionalisierter politischer Inhalte geben. Doch das alles ist doch auch im Bereich professioneller Politik seit je der Fall. Also wird dergleichen für ein Problem nur jener halten, der mit plebiszitären Instrumenten die Welt realer Politik zu überwinden hofft.

Weil sich das aber ganz gewiss nicht ereignen wird, muss man auf die Finanzierungspraktiken von Gruppierungen, die sich dem Einsatz plebiszitärer Instrumente verschreiben, ebenso scharf wie bei den politischen Parteien achten. Das gilt umso mehr, als gerade der punktuelle Charakter plebiszitärer Kampagnen organisierte Interessen und ihnen nahestehende Kapitalgeber anziehen wird. Nicht nur die Bürgerschaft, sondern auch organisations- und konfliktfähige Lobbygruppen werden also von einem Ausbau plebiszitärer Instrumente profitieren. Gegen deren verhohlene Vermachtung, ja auch Vermarktung demokratischer Willensbildung und Entscheidungsfindung im Wege des Plebiszits muss man deshalb wirkungsvolle gesetzliche Regelungen vorsehen. Man darf sich aber auch nicht wundern, wenn diese Regeln im Einzelfall ebenso kunstvoll umgangen werden, wie das Parteien bei ihrer eigenen Finanzierung immer schon tun. Gerade hier sind kritische Massenmedien gefragt, die sich dann allerdings nicht länger anwaltschaftlich in den Dienst einzelner Initiatorengruppen stellen dürften, sondern - wie bei Wahlkämpfen - gerade auch deren Hintergründe und Niederungen auszuleuchten hätten.

Viel wichtiger als die zu erwartenden, durchaus auch unschönen Begleiterscheinungen plebiszitärer Instrumente ist aber, dass nach deren Einführung Behauptungen leicht überprüfbar würden, hinter lautstarkem Bürgerprotest stünde auch eine Mehrheit der Bürger. Ganz gleich welches der vorgeschlagenen plebiszitären Instrumente man auch nutzt: Am Ende ist immer klar, wie viele Bürger sich für welche Position wirklich mobilisieren ließen, und wie die realen, also in einer Demokratie dann auch zu akzeptierenden, Mehrheitsverhältnisse sind. Gewöhnt sich nach einiger Zeit das Volk - und vor allem: die jetzt schon unübersehbare Landsknechtstruppe der „Protestindustrie“ - daran, Niederlagen bei Volksabstimmungen ebenso zu akzeptieren wie Niederlagen bei Wahlen, dann dürften zumal plebiszitäre Instrumente jene befriedende - und dann auch: die Umsetzung von Entscheidungen beschleunigende - Kraft entfalten, die man derzeit angesichts zunehmender Bürgerproteste weithin vermisst. Immerhin kann sich eine plebiszitär unterlegene Gruppe nicht mehr plausibel darauf berufen, für die Interessen der Mehrheit oder gar „für mehr Demokratie“ zu kämpfen. Das aber sollte, eine wirklich demokratisch gesinnte Bürgerschaft vorausgesetzt, ihren Zulauf stoppen und die Quellen ihrer Ressourcen versiegen lassen. Tatsäch- 
lich ist, und ganz anders als oft befürchtet, beim Einsatz der empfohlenen plebiszitären Instrumente gerade keine „Herrschaft der Minderheit über die Mehrheit“ zu befürchten: Beim Volksentscheid gewinnt immer die artikulationswillige Mehrheit. Nur muss sich schon die Mühe eigener politischer Beteiligung machen, wer sich durchsetzen will. Tatsächlich wird auch bei Wahlen mit einer Wahlbeteiligung unter 50 Prozent nicht auf die durch Wahlabstinenz „schweigende Mehrheit“ gehört, sondern wird ganz entschieden die Behauptung zurückgewiesen, eine auf zwar die Mehrheit der Parlamentssitze gegründete, doch nur von einer Minderheit der Wahlberechtigten getragene Regierung erfülle den Tatbestand einer „Minderheitsherrschaft“.

Noch folgenreicher und willkommener mag sein, dass die vorgeschlagenen plebiszitären Instrumente - einmal eingeführt - es weniger wahrscheinlich machen, dass sich pluralistische Konflikte zwischen politischen Lagern, die unvereinbare Ziele anstreben, weiterhin wie Konflikte zwischen der regierenden Mehrheit als „dem Staat“ und dem ,(vergeblich) protestierenden Volk“ ausprägen. Nichts untergräbt nämlich mehr als diese teils inszenierte, teile realisierte Frontstellung die demokratische Legitimität unseres Gemeinwesens. Bislang jedenfalls neigt bei weltanschaulich stark aufgeladenen Konflikten - früher: für oder gegen Atomwaffen, später: für oder gegen Atomenergie - die bei Wahlen oder im Gesetzgebungsverfahren unterlegene Minderheit dazu, nach Ausschöpfung auch ihrer verfassungs- und verwaltungsgerichtlichen Möglichkeiten unter dem Feldzeichen der Demokratie mit öffentlichen Protesten und zivilem Ungehorsam gegen jene Mehrheit vorzugehen, welche nun einmal den Staat regiert und dessen Zwangsmittel einsetzen kann. Doch der Glaube an die bislang immer recht erfolgreich beanspruchte demokratische Legitimität vor allem zivilen Ungehorsams dürfte rasch schwinden, wenn statt eines Konflikts zwischen „Staat“ und ,außerparlamentarischer Opposition“ einfach einer zwischen plebiszitär konkurrierenden Gruppen ausgetragen wird, bei dem es faktisch nebensächlich ist, welche dieser Gruppen gerade (mit-) regiert. Im Anschluss an einen plebiszitären Sieg der vormaligen Minderheit gibt es für diese nämlich keine Notwendigkeit mehr für Protest und zivilen Ungehorsam, während umgekehrt im Anschluss an eine plebiszitäre Niederlage das legitimatorische Argument der nun offensichtlich gewordenen Minderheit entfällt, man vertrete doch die Interessen der bislang sprachlos gehaltenen Mehrheit.

Und ohnehin entfalten plebiszitäre Instrumente ihren bestmöglichen Nutzen für die repräsentative Demokratie gerade dann, wenn sie überhaupt Teil einer breiteren Palette praktizierter Bürgerbeteiligung sind. Gewiss sind die dafür jetzt schon verfügbaren Möglichkeiten - von dauerhafter politischer Tätigkeit in Parteien und Verbänden über projektbezogenes Engagement in Bürgerinitiativen bis 
zur fallweisen Teilnahme an Demonstrationen - keine geringen Errungenschaften und durchaus wirkungsvoll. Allerdings wird den wachsenden Ansprüchen zumal gebildeter und so gut situierter Bürger, dass sie auch für die Politik leben können, auf zusätzliche Foren und Wege politischer Partizipation noch nicht ausreichend Rechnung getragen. Dabei sind die grundsätzlich verfügbaren Möglichkeiten doch längst bekannt und müssten nur praktisch mehr genutzt werden: von E-Partizipation über Bürgerhaushalte bis hin zu Planungszellen und Perspektiven- bzw. Zukunftswerkstätten. Erfüllten wir das alles mit politischem Leben und legten wir uns außerdem die vorgeschlagenen plebiszitären Instrumente bereit: Wir stünden dann zwar immer noch nicht vor einer ,,vollkommenen Demokratie“, doch gewiss am Ende jener Zeit, in der man glaubwürdig über ,unvollendete Demokratie“ lamentieren und politische Abstinenz durch angebliche Aussichtslosigkeit bürgergesellschaftlichen Engagements rechtfertigen konnte. Allein schon das wäre ein großer Fortschritt. 